\title{
Molecular mechanisms of ATP secretion during immunogenic cell death
}

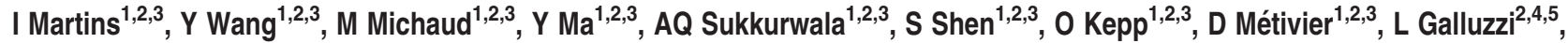 \\ J-L Perfettini ${ }^{1,2,3}$, L Zitvogel ${ }^{2,3,6,7}$ and G Kroemer ${ }^{\star, 1,4,5,8,9}$
}

The immunogenic demise of cancer cells can be induced by various chemotherapeutics, such as anthracyclines and oxaliplatin, and provokes an immune response against tumor-associated antigens. Thus, immunogenic cell death (ICD)-inducing antineoplastic agents stimulate a tumor-specific immune response that determines the long-term success of therapy. The release of ATP from dying cells constitutes one of the three major hallmarks of ICD and occurs independently of the two others, namely, the pre-apoptotic exposure of calreticulin on the cell surface and the postmortem release of high-mobility group box 1 (HMBG1) into the extracellular space. Pre-mortem autophagy is known to be required for the ICD-associated secretion of ATP, implying that autophagy-deficient cancer cells fail to elicit therapy-relevant immune responses in vivo. However, the precise molecular mechanisms whereby ATP is actively secreted in the course of ICD remain elusive. Using a combination of pharmacological screens, silencing experiments and techniques to monitor the subcellular localization of ATP, we show here that, in response to ICD inducers, ATP redistributes from lysosomes to autolysosomes and is secreted by a mechanism that requires the lysosomal protein LAMP1, which translocates to the plasma membrane in a strictly caspase-dependent manner. The secretion of ATP additionally involves the caspase-dependent activation of Rho-associated, coiled-coil containing protein kinase 1 (ROCK1)-mediated, myosin II-dependent cellular blebbing, as well as the opening of pannexin 1 (PANX1) channels, which is also triggered by caspases. Of note, although autophagy and LAMP1 fail to influence PANX1 channel opening, PANX1 is required for the ICD-associated translocation of LAMP1 to the plasma membrane. Altogether, these findings suggest that caspase- and PANX1-dependent lysosomal exocytosis has an essential role in ATP release as triggered by immunogenic chemotherapy.

Cell Death and Differentiation (2014) 21, 79-91; doi:10.1038/cdd.2013.75; published online 12 July 2013

The field of anticancer therapy has recently witnessed a significant paradigm shift. For a long time, cytotoxic antineoplastic agents were assumed to mediate therapeutic effects owing to their capacity to directly kill malignant cells or to induce their senescence. Accumulating evidence now indicates that (at least some) anticancer agents actively stimulate tumor-specific immune responses, which in many cases account for long-term therapeutic successes. ${ }^{1-4}$ Chemotherapy (as well as some forms of radiotherapy) can exert immunostimulatory effects via two alternative, although non-exclusive, mechanisms. First, some cytotoxic anticancer agents, including anthracyclines and oxaliplatin (OXA), are capable of triggering an immunogenic variant of apoptosis known as immunogenic cell death (ICD), ${ }^{5,6}$ de facto converting dying cancer cells into a therapeutic vaccine..$^{7,8}$ Second, multiple chemotherapeutics can directly stimulate antitumor immunity, ${ }^{1,4}$ either by potentiating the activity of immune effectors (e.g., vinca alkaloids have been shown to promote the maturation of dendritic cells (DCs)) or by antagonizing immunosuppressive cells (e.g., cyclophosphamide reportedly depletes/inhibits regulatory T cells). ${ }^{9,10}$

ICD has been operatively defined as a cell death modality that elicits a protective immune response against dead-cell antigens, implying that the immunogenicity of cell death can be monitored in appropriate vaccination assays. ${ }^{2,11,12}$ Thus, the subcutaneous injection of cancer cells that are succumbing to ICD, but not of cells undergoing conventional apoptosis or necrosis, elicits a T-cell-mediated immune response protecting histocompatible mice against a subsequent challenge with tumor cells of the same type. ${ }^{2,3,13}$ Of note, most inducers of apoptosis and necrosis fail to trigger ICD. However, a few chemotherapeutics, including

\footnotetext{
${ }^{1}$ INSERM, U848, F-94805 Villejuif, France; ${ }^{2}$ Institut Gustave Roussy, F-94805 Villejuif, France; ${ }^{3}$ Université Paris Sud/Paris XI, F-94270 Le Kremlin Bicêtre, France; ${ }^{4}$ Université Paris Descartes/Paris V, Sorbonne Paris Cité, F-75006 Paris, France; ${ }^{5}$ Equipe 11 labellisée par la Ligue Nationale contre le Cancer, Centre de Recherche des Cordeliers, F-75006 Paris, France; ${ }^{6}$ INSERM, U1015 labellisée par la Ligue Nationale contre le Cancer, F-94805 Villejuif, France; ${ }^{7}$ Center of Clinical Investigations CBT507, Institut Gustave Roussy, F-94805 Villejuif, France; ${ }^{8}$ Metabolomic and Cell Biology Platforms, Institut Gustave Roussy, F-94805 Villejuif, France and ${ }^{9}$ Pôle de Biologie, Hôpital Européen Georges Pompidou, AP-HP, F-75015 Paris, France

*Corresponding author: G Kroemer, INSERM U848, Institut Gustave Roussy, 114, rue Edouard Vaillant, F-94805 Villejuif, France. Tel: + 33142116046 ; Fax: + 33142116047 ; E-mail: kroemer@orange.fr

Keywords: apoptosis; Beclin 1; caspases; endoplasmic reticulum stress; quinacrine; U2OS cells

Abbreviations: BCN1, Beclin 1; CRT, calreticulin; $\Delta \psi_{m}$, mitochondrial transmembrane potential; DIDS, 4,4'-diisothiocyano-2,2'-stilbenedisulfonic acid; HMGB1, highmobility group box 1; ICD, immunogenic cell death; FBS, fetal bovine serum; FITC, fluorescein isothiocyanate; GFP, green fluorescent protein; MTX, mitoxantrone; PANX1, pannexin 1; PE, phycoerythrin; PI, propidium iodide; PS, phosphatidylserine; RFP, red fluorescent protein; ROCK1, Rho-associated, coiled-coil containing protein kinase 1; siRNA, small-interfering RNA; SITS, 4-acetamido-4-isothiocyano-stilbene-2,2-disulfonic acid; SNARE, SNAP receptor; TMRM, tetramethylrhodamine methyl ester; tPANX1, truncated PANX1; VAMP1, vesicle-associated membrane protein 1; VNUT, vesicular nucleotide transporter

Received 8.2.13; revised 29.5.13; accepted 31.5.13; Edited by M Piacentini; published online 12.7.13
} 
anthracyclines, ${ }^{7,8}$ OXA, ${ }^{14}$ cyclophosphamide, ${ }^{15}$ and - to some extent - microtubular inhibitors, ${ }^{16}$ as well as cardiac glycosides, ${ }^{17-19}$ potently do so. ${ }^{20,21}$ Such chemicals appear to be particularly efficient at inducing a pre-mortem endoplasmic reticulum (ER) stress response and autophagy. ER stress culminates in the translocation of the ER chaperone calreticulin (CRT) to the cell surface, thereby generating an 'eat-me' signal for DCs. ${ }^{3,22}$ Autophagy facilitates the release of ATP from dying cells, ${ }^{23}$ constituting both a 'find-me' signal for the recruitment of DCs and their precursors ${ }^{24}$ and a proinflammatory stimulus that - upon binding to the purinergic receptor $\mathrm{P} 2 \mathrm{RX} 7$ - elicits the activation of the NOD-like receptor family, pyrin domain containing 3 (NLRP3) inflammasome within DCs and macrophages. ${ }^{25,26}$ In addition, ICD is associated with the postmortem release of the non-histone chromatin-binding protein high-mobility group box 1 (HMGB1) into the extracellular space, allowing HMGB1 to bind Toll-like receptor 4 on DCs and thus stimulate their antigenpresenting functions. ${ }^{2,27}$

CRT exposure, ATP secretion and HMGB1 release are all indispensable for ICD, meaning that the absence of one single of these ICD hallmarks abolishes the efficacy of anthracycline- or OXA-based chemotherapy in mouse models. ${ }^{2}$ For example, the transgene-driven overexpression of the ectonucleotidase CD39, which converts extracellular ATP into ADP and AMP, by tumor cells is sufficient to compromise the therapeutic effects of ICD-inducing antineoplastic agents in vivo. $^{28}$ Along similar lines, the stable knockdown of essential autophagic factors such as ATG5 and ATG7, which inhibits ATP secretion from dying tumor cells, allows for the generation of neoplastic lesions that fail to respond to ICD inducers. $^{23}$

The precise molecular mechanisms whereby ICD inducers stimulate the active secretion of ATP by dying cells are elusive. Malignant cells secrete ATP in response to a large panel of cytotoxic therapeutics, ${ }^{23,29}$ and this phenomenon obligatorily involves the pre-mortem activation of the autophagic machinery. ${ }^{23}$ Although the induction of autophagy alone (in the absence of cell death) may result per se in the secretion of ATP, ${ }^{30}$ significantly higher extracellular ATP levels are achieved when autophagy and cell death concur. ${ }^{23,25}$ Pannexin 1 (PANX1) channels are known to have a prominent role in the release of ATP from apoptotic cells. Indeed, caspase 3 , which is a major factor in the execution of apoptotic cell death, ${ }^{5,6}$ cleaves PANX1 at its C-terminal auto-inhibitory domain, thereby generating a truncated form of the protein (tPANX1) that operates as a constitutively active channel. ${ }^{31}$ In line with this notion, the pharmacological inhibition of caspases, the knockout of Panx1, the depletion of PANX1 as well as the substitution of PANX1 by a non-cleavable variant all abolish apoptosis-associated ATP release. ${ }^{32-34}$ Moreover, caspase activation and PANX1 expression are both required for the partial, apoptotic increase in the permeability of the plasma membrane to small solutes that precedes the complete, post-apoptotic (necrotic) plasma membrane breakdown. ${ }^{32-34}$ In a cell death-unrelated setting, astrocytes have been reported to secrete ATP by lysosomal exocytosis, a process in which lysosomes fuse with the plasma membrane to release their luminal content into the extracellular space. ${ }^{35}$
Driven by these premises and incognita, we decided to characterize the mechanisms through which cancer cells extrude ATP in response to chemotherapeutic agents. Here, we demonstrate that the secretion of ATP by dying cancer cells obligatorily involves multiple signal transduction pathways that are articulated in a complex manner.

\section{Results}

Involvement of autophagy and caspases in ICDassociated ATP secretion. Live human osteosarcoma U2OS cells maintained in control conditions and stained with the ATP-sensitive fluorochrome quinacrine, which is usually employed for the detection of intracellular ATP-containing vesicles, ${ }^{36}$ exhibit a green fluorescence that can readily be monitored by cytofluorometry. This signal is significantly reduced when U2OS cells are co-treated with 2-deoxyglucose (an inhibitor of glycolysis) and antimycin A (which blocks mitochondrial respiration at the level of complex III; Figure 1a), reflecting a significant depletion of intracellular ATP stores. Chemotherapeutics such as mitoxantrone (MTX) and OXA induced a dose-dependent reduction in the amount of ATP that was detectable with quinacrine in viable $\left(4^{\prime}, 6\right.$-diaminidino-2phenylindole $(\mathrm{DAPI})^{-}$) U2OS cells, even before they exposed phosphatidylserine (PS) on their surface and thus became able to bind phycoerythrin (PE)-conjugated AnnexinV (Figures 1a and b). The co-staining of MTX-treated U2OS cells with a mitochondrial transmembrane potential $\left(\Delta \psi_{\mathrm{m}}\right)$-sensitive probe and PE-conjugated AnnexinV confirmed that intracellular ATP levels drop along with the induction of apoptosis and that cells that exhibit a reduced $\Delta \psi_{\mathrm{m}}$, but have not yet exposed PS, are particularly prone to secrete ATP into the extracellular milieu when re-placed in culture (Figures $1 c$ and d). Of note, MTXinduced ATP depletion was strongly inhibited by the broadspectrum caspase inhibitor Z-VAD-fmk (Figure 1b). In line with previous reports, ${ }^{23,37}$ autophagy inhibition also compromised the ability of U2OS cells to secrete ATP (Figure 1e), but not to die (Supplementary Figure 1), in response to MTX. The inhibition of autophagy blocked ATP secretion by MTX-treated cells both in the absence and presence of the ecto-ATPase inhibitor ARL67156, excluding the possibility that the overexpression of ecto-ATPases by autophagy-deficient cells would account for these observations (Figure 1e). Of note, autophagy inhibition was not accompanied by a reduction of intracellular ATP levels in baseline conditions (Supplementary Figure 2). Moreover, rapamycin, spermidine and resveratrol, which potently stimulate autophagy but fail to induce cell death (Supplementary Figure 1 and data not shown), were all unable to induce significant ATP secretion (Figure 1e and data not shown).

Taken together, these results indicate that autophagy is required - but not sufficient - for the ICD-associated release of ATP. Rather, it appears that only the combination of autophagy and apoptosis-related processes (such as caspase activation) allows for ATP secretion in the course of ICD.

Involvement of Rho-associated, coiled-coil containing protein kinase 1 (ROCK1) and PANX1 channels in ICD-associated ATP release. To gain further insights into the mechanisms whereby some (but not all) chemo- 
a

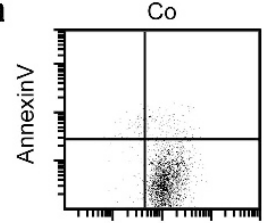

b
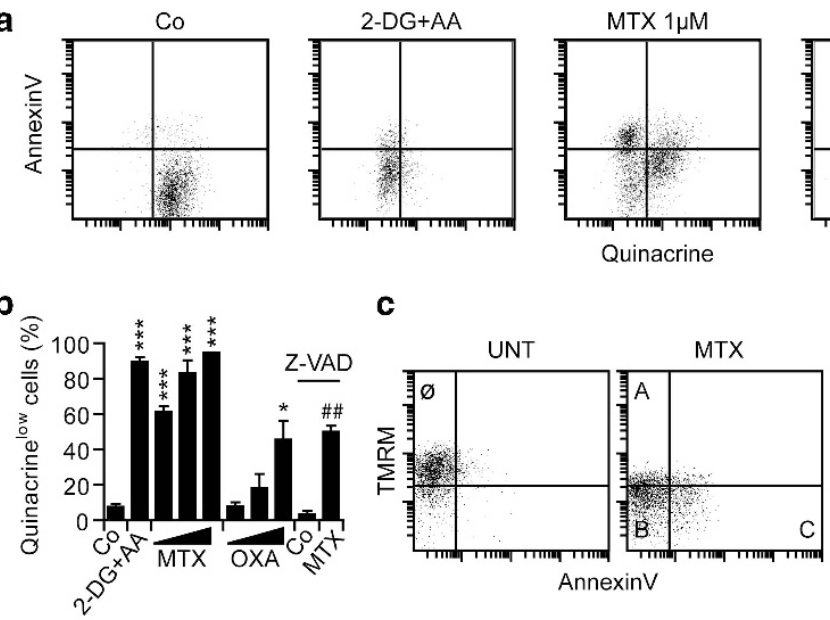

Quinacrine

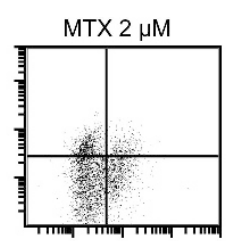

d

e
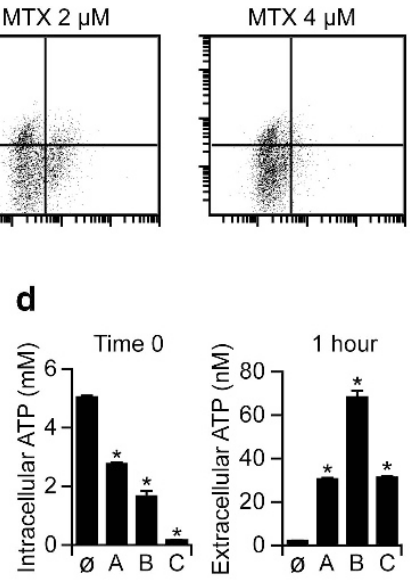

$\mathrm{CR}$

ATG5 ${ }^{\mathrm{KD}}$

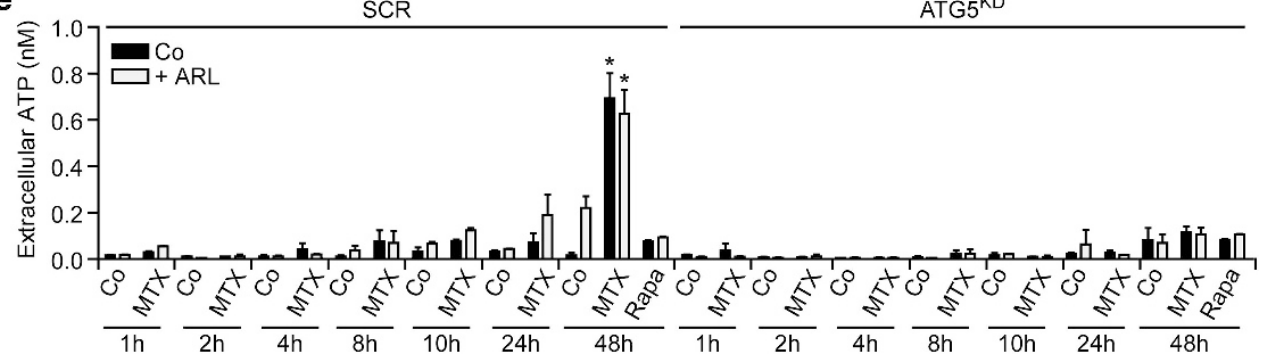

Figure 1 Intra- and extracellular ATP levels in the course of immunogenic cell death. (a and $\mathbf{b}$ ) Human osteosarcoma U2OS cells were maintained in control conditions (Co) or treated with $30 \mathrm{mM}$ 2-deoxyglucose (2-DG), $2 \mu \mathrm{g} / \mathrm{ml}$ antimycin A (AA), 150, 300 or $600 \mu \mathrm{M}$ oxaliplatin (OXA) or 1, 2 or $4 \mu \mathrm{M}$ mitoxantrone (MTX, $4 \mu \mathrm{M}$ when not otherwise indicated), alone or combined with $50 \mu \mathrm{M} Z$ Z-VAD-fmk (Z-VAD) for $24 \mathrm{~h}$, and then co-stained for the cytofluorometric detection of intracellular ATP (with quinacrine, green fluorescence) and phosphatidylserine exposure (with PE-conjugated AnnexinV, red fluorescence). Representative dot plots and quantitative data (means \pm S.E.M., $n=3$ ) are reported. ${ }^{*} P<0.05,{ }^{* \star *} P<0.001$ (Student's $t$-test), as compared with cells maintained in Co conditions; ${ }^{\# \#} P<0.01$ (Student's $t$-test), as compared with cells treated with $4 \mu \mathrm{M}$ MTX only. (c and d) U2OS cells were maintained in Co conditions or treated with $4 \mu \mathrm{M}$ MTX for $18 \mathrm{~h}$, and then co-stained for the cytofluorometric detection of plasma membrane integrity (with DAPI, blue fluorescence), mitochondrial transmembrane potential (with TMRM, red fluorescence) and phosphatidylserine exposure (with FITCconjugated AnnexinV, green fluorescence). DAPI ${ }^{-}$cells were then sorted according to TMRM and FITC signals, as indicated (c) and either immediately lysed for the assessment of intracellular ATP levels or re-placed in culture for $1 \mathrm{~h}$, followed by the quantification of ATP secretion in culture supernatants (d). Representative dot plots and quantitative data (means \pm S.E.M., $n=3$ ) are reported. ${ }^{*} P<0.05$ (Student's $t$-test) as compared with cells in gate $\varnothing$. (e) Murine fibrosarcoma MCA205 cells stably transfected with either a scrambled (SCR) or an Atg5-targeting shRNA (Atg $5^{\mathrm{KD}}$ ) were maintained in Co conditions or treated with $4 \mu \mathrm{M}$ MTX alone or in combination with $3 \mu \mathrm{M}$ ARL67156 (ARL) for the indicated time, followed by the assessment of ATP secretion into culture supernatants. Quantitative data (means \pm S.E.M., $n=3$ ) are reported. ${ }^{*} P<0.05$ (Student's $t$-test) as compared with cells maintained in Co conditions or treated with ARL only for $48 \mathrm{~h}$

therapeutics induce the release of ATP by cancer cells as they kill them, we performed a chemical screen. Thus, U2OS cells were treated with 1530 distinct chemicals from the ICCB Known Bioactives Library or the US-Drug Collection (encompassing most US Food and Drug Administration-approved and some experimental drugs), alone or in combination with MTX, for $18 \mathrm{~h}$, stained with quinacrine and then analyzed by automated, quantitative epifluorescence microscopy (Figure 2a). This screen led to the identification of several compounds that are capable of preventing the MTX-triggered loss of quinacrine-dependent fluorescence (Figure 2b): monensin, which blocks intracellular trafficking and exocytosis; ${ }^{38}$ blebbistatin, which inhibits myosin II, thereby blocking the apoptosis-associated blebbing of the plasma membrane; ${ }^{39}$ Y-27632, which inhibits ROCK1, ${ }^{40}$ a protein that - upon cleavage by caspase-3 - participates in apoptotic blebbing; ${ }^{41,42}$ and mefloquine, which inhibits PANX1 channels. ${ }^{43}$ Subsequent validation experiments confirmed that monensin, blebbistatin and several chemically-unrelated inhibitors of ROCK1 (i.e., Y-27632, bearing with a central aminoethyl group, and $\mathrm{H}-1152$, characterized by a central sulfonyl junction) and PANX1 (i.e., the synthetic quinine analogue mefloquine and the disulfonic stilbene derivatives 4,4'-diisothiocyano-2,2' -stilbenedisulfonic acid (DIDS) and 4-acetamido-4-isothiocyano-stilbene-2,2-disulfonic acid (SITS)) blunt the loss of quinacrine fluorescence as triggered in U2OS cells by MTX (Figure 2c). Similar results were obtained when intracellular ATP levels were measured by means of a commercial, luciferase-based kit (Figure 2d) as well as by an alternative ATP-sensitive fluorochrome, Bodipy-ATP ${ }^{38}$ (Supplementary Figure 3). In addition, the ICD-associated release of ATP was inhibited by a panel of distinct pannexin (and connexin) inhibitors (Supplementary Figure 4). Finally, the small-interfering RNA (siRNA)mediated depletion of ROCK1 or PANX1 as well as that of SLC17A9, a protein involved in the exocytosis of ATPcontaining vesicles (also known as vesicular nucleotide transporter, VNUT), ${ }^{44}$ inhibited ATP secretion by U2OS cells 
a

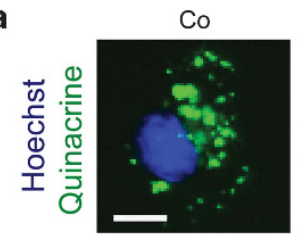

b
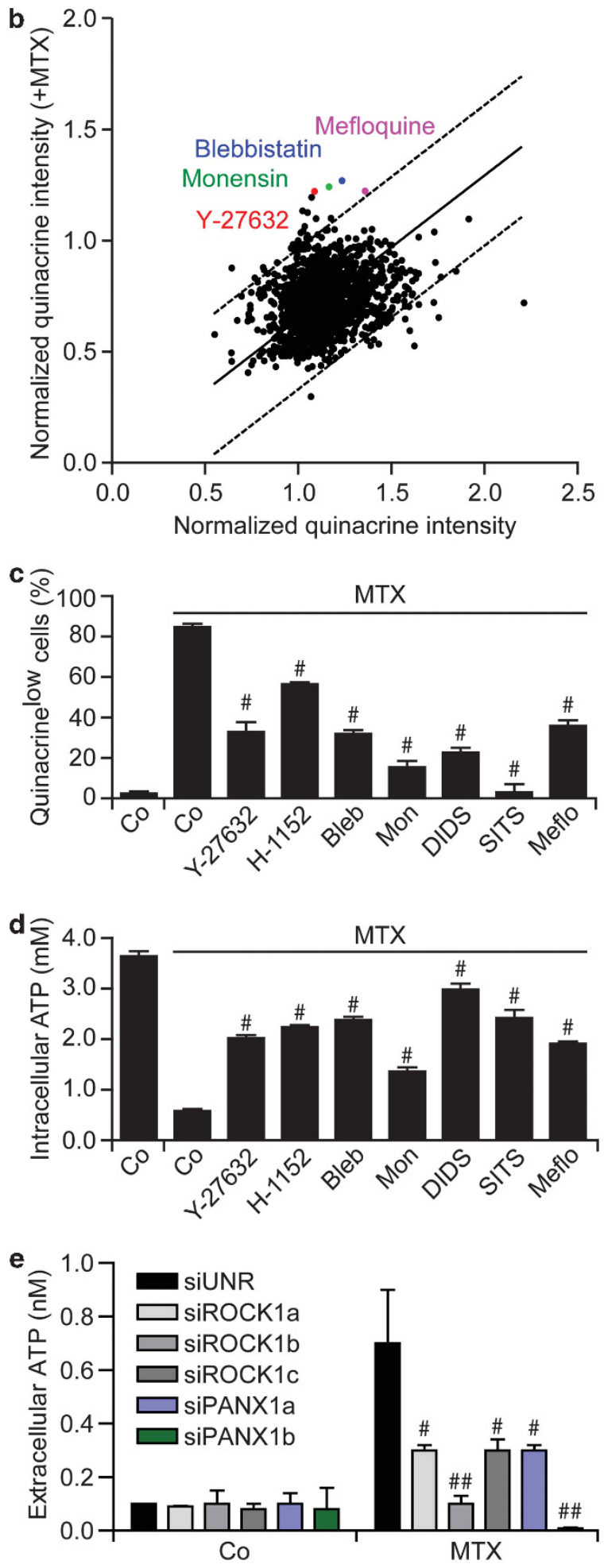

responding to MTX (Figure 2e, Supplementary Figure 5), confirming the results obtained with pharmacological inhibitors.

Although pharmacological inhibitors of ROCK1, PANX1 or myosin II interfered with ATP release as induced by OXA, they failed to prevent OXA-triggered apoptosis and were unable to interfere with the pre-apoptotic exposure of CRT on the cell surface and the post-apoptotic release of HGMB1 associated with ICD (Supplementary Figure 6). Moreover, ROCK1, PANX1 and myosin II inhibitors failed to alter autophagy, both in baseline conditions and following the administration of OXA (Supplementary Figure 6), suggesting that these factors act downstream of autophagy to mediate the ICD-associated release of ATP. Importantly, the inhibition of ROCK1, PANX1 or myosin II suppressed the capacity of MTX to elicit ICD, as evaluated in vaccination experiments. Thus, the subcutaneous administration of CT26 colon carcinoma and MCA205 fibrosarcoma cells treated with MTX alone did protect haplocompatible $\mathrm{BALB} / \mathrm{c}$ and $\mathrm{C} 57 \mathrm{BI} / 6$ mice, respectively, against a subsequent challenge with live tumor cells of the same type (Supplementary Figure 7). However, CT26 and MCA205 cells succumbing to MTX in the presence of ROCK1, PANX1 or myosin II inhibitors became unable to successfully vaccinate mice against cancer (Supplementary Figure 7).

Collectively, these data point to an essential role for both apoptotic blebbing (which depends on ROCK1 and myosin II) and PANX1 channels in ICD-associated ATP release.

PANX1 channels operate independently from autophagy. A panel of distinct chemotherapeutic agents provoked the translocation of PANX1 to the outer leaflet of the plasma membrane, rendering it detectable by immunofluorescence microscopy upon cell surface staining. Thus, although untreated U2OS cells failed to stain positively for cell surface PANX1, chemotherapy-treated cells exhibited a punctuate PANX1specific staining, a phenomenon that was completely suppressed by Z-VAD-fmk (Figures $3 a$ and b). Next, we generated MCA205 cells that express tPANX1 under the control of the

Figure 2 Chemical library screen for the identification of ATP release inhibitors. (a and b) Human osteosarcoma U2OS cells were treated with 1530 compounds from the ICCB Known Bioactives Library (final concentration $30 \mu \mathrm{M}$ ) or the US-Drug collection (final concentration $1 \mu \mathrm{M}$ ), alone or combined with $4 \mu \mathrm{M}$ mitoxantrone (MTX), for $18 \mathrm{~h}$, and then stained for the fluorescence microscopy-assisted detection of nuclei (with Hoechst 33342, blue fluorescence) and ATP-containing vesicles (with quinacrine, green fluorescence). Representative images (scale bar $=10 \mu \mathrm{m})$ and quantitative data are reported. Each dot represents the normalized mean quinacrine fluorescence associated with one compound alone ( $x$ axis) and combined with MTX ( $y$ axis). (c and d) U2OS cells were maintained in control (Co) conditions or treated with $60 \mu \mathrm{M} \mathrm{Y}-27632,2 \mu \mathrm{M} \mathrm{H}-1152,25 \mu \mathrm{M}$ blebbistatin (Bleb), $5 \mu \mathrm{M}$ monensin (Mon), $10 \mu \mathrm{M}$ 4,4'-diisothiocyano-2,2'stilbenedisulfonic acid (DIDS), $10 \mu \mathrm{M}$ 4-acetamido-4-isothiocyano-stilbene-2,2disulfonic acid (SITS) and $10 \mu \mathrm{M}$ mefloquine (Meflo), alone or combined with $4 \mu \mathrm{M}$ MTX, for $18 \mathrm{~h}$, and then stained for the cytofluorometric (c) or luminometric (d) quantification of ATP levels. Quantitative data (means \pm S.E.M., $n=3$ ) are reported. ${ }^{\#} P<0.05$ (Student's $t$-test) as compared with cells treated with MTX only. (e) U2OS cells were transfected with a non-targeting siRNA (siUNR) or with the indicated siRNAs for $48 \mathrm{~h}$, and then maintained in Co conditions or treated with $4 \mu \mathrm{M}$ MTX for additional $18 \mathrm{~h}$. Eventually, ATP release in culture supernatants was monitored by a luciferase-based test. ${ }^{\#} P<0.05,{ }^{\# \#} P<0.01$ (Student's $t$-test) as compared with siUNR-transfected cells treated with MTX only 
a

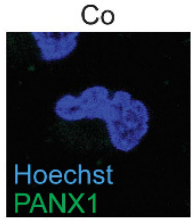

Z-VAD
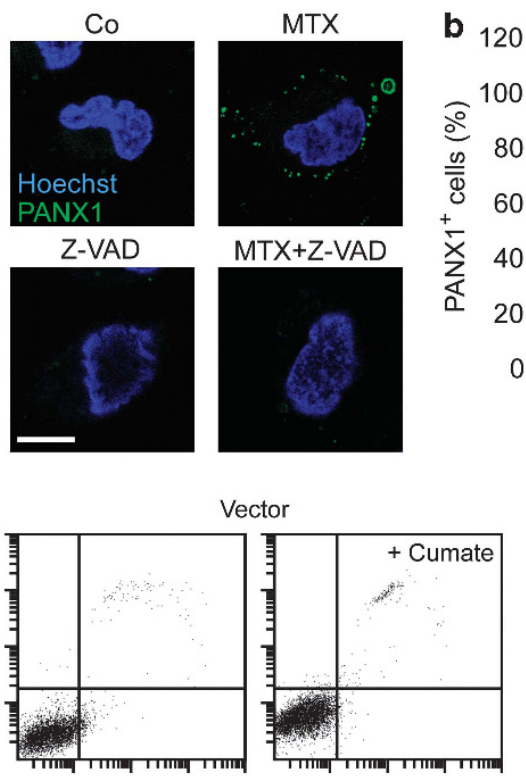

YO-PRO ${ }^{\circledR}-1$
Vector

C
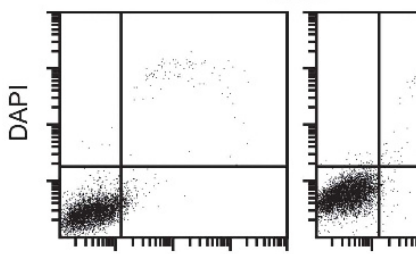

Co

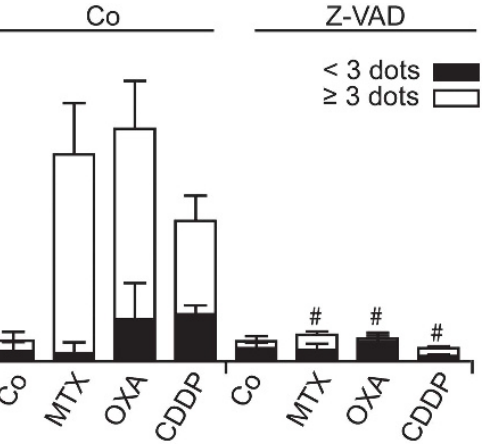

d
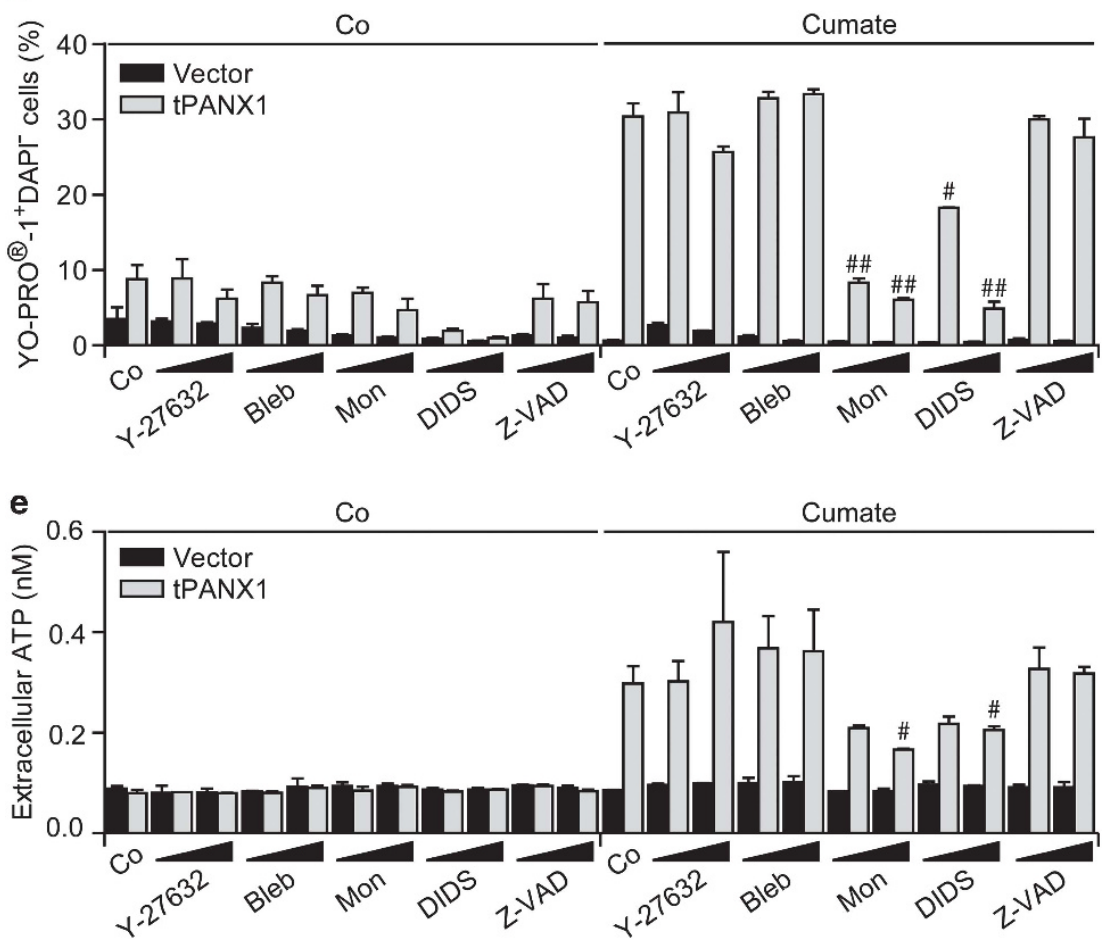

Figure 3 ATP release through PANX1 channels. (a and $\mathbf{b})$ Human osteosarcoma U2OS cells were maintained in control conditions (Co) or treated with $2 \mu \mathrm{M}$ mitoxantrone (MTX), $300 \mu \mathrm{M}$ oxaliplatin (OXA) and $75 \mu \mathrm{M}$ cisplatin (CDDP), alone or in combination with $50 \mu \mathrm{M}$ Z-VAD-fmk (Z-VAD-fmk), for $18 \mathrm{~h}$, and stained for the fluorescence microscopy-assisted detection of nuclei (with Hoechst 33342, blue fluorescence) and PANX1 at the cell surface (green fluorescence). Representative images (scale $\mathrm{bar}=10 \mu \mathrm{m}$ ) and the $\%$ of cells exhibiting $<3$ or $\geq 3$ PANX1 dots are reported (means \pm S.E.M., $n>100$ cells). ${ }^{\#} P<0.05$ (Student's $t$-test) as compared with cells treated with MTX, OXA or CDDP only. (c-e) Murine fibrosarcoma MCA205 cells stably transfected with a construct coding for truncated PANX1 (tPANX1) under the control of a cumate-inducible promoter or with the corresponding empty vector were maintained in Co conditions or treated with 15 or $30 \mu \mathrm{M} \mathrm{Y}-27632,5$ or $10 \mu \mathrm{M}$ blebbistatin (Bleb), 2.5 or

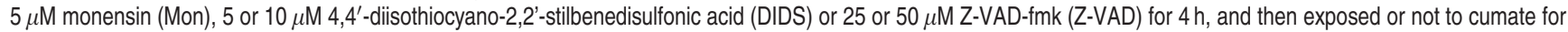
additional $48 \mathrm{~h}$. Finally, cells were processed for the cytofluorometric detection of YO-PRO-1 and DAPI uptake (c and d) and ATP concentration in supernatants was assayed by a luciferase-based assay (e). Representative dot plots and quantitative data (means \pm S.E.M., $n=3$ ) are reported. ${ }^{\#} P<0.05,{ }^{\#} P<0.01$ (Student's $t$-test) as compared with cells treated with cumate only 

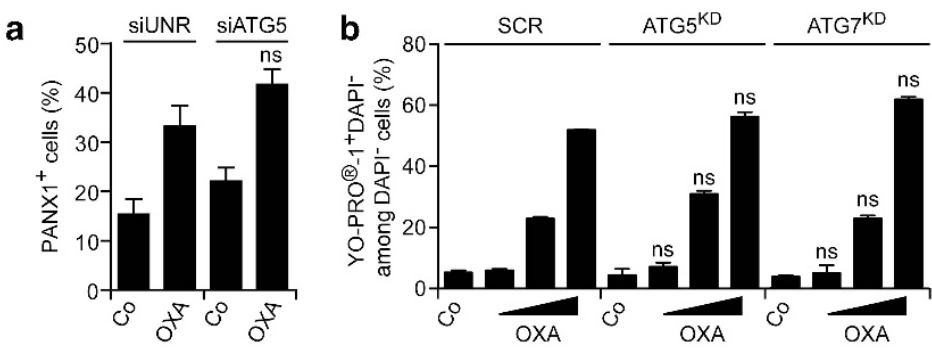

c
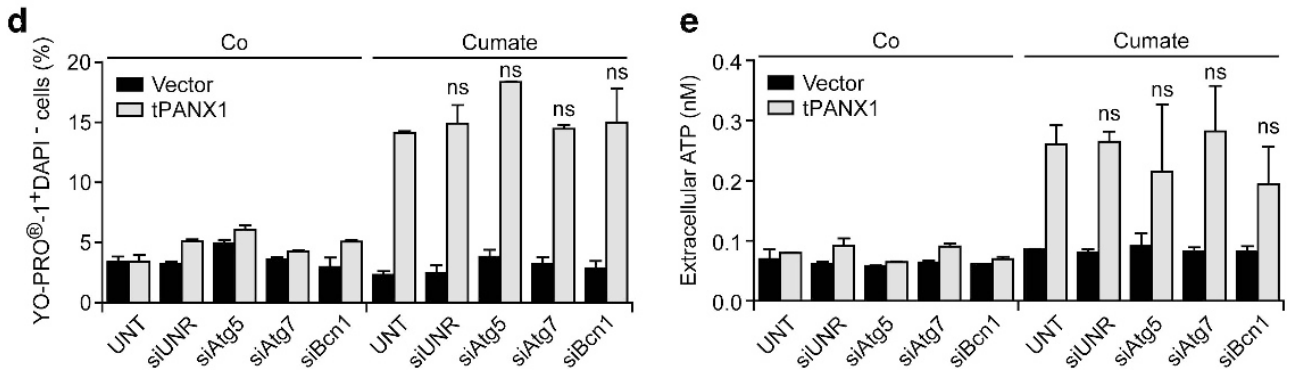

Figure 4 Autophagy-independent ATP release through PANX1 channels. (a) Human osteosarcoma U2OS cells were transfected with a non-targeting siRNA (siUNR) or with a siRNA specific for ATG5 for $48 \mathrm{~h}$, then maintained in control (Co) conditions or treated with $300 \mu \mathrm{M}$ oxaliplatin (OXA) for additional $18 \mathrm{~h}$. Finally, cells were processed for the immunofluorescence microscopy-assisted detection of PANX1 at the cell surface. The percentage of cells exhibiting $\geq 3$ PANX1 dots are reported (means \pm S.E.M., $n>100$ cells). ns, non-significant (Student's t-test) as compared with siUNR-transfected, OXA-treated cells. (b) Murine fibrosarcoma MCA205 cells stably transfected with either a scrambled (SCR) shRNA or shRNAs targeting Atg5 (Atg $5^{\mathrm{KD}}$ ) or $\operatorname{Atg} 7\left(A \operatorname{tg} 7^{\mathrm{KD}}\right.$ ) were maintained in Co conditions or treated with 75,150 or $300 \mu \mathrm{M}$ OXA for $18 \mathrm{~h}$, followed by the cytofluorometric detection of YO-PRO-1 and DAPI uptake. Quantitative data are reported (means \pm S.E.M., $n=3$ ). ns, non-significant (Student's $t$ test) as compared with equally treated SCR cells. (c) Murine fibrosarcoma MCA205 cells stably transfected with a construct coding for truncated PANX1 (tPANX1) under the control of a cumate-inducible promoter or with the corresponding empty vector were treated or not with cumate $(\mathrm{Cu})$ for $48 \mathrm{~h}$, followed by the immunoblotting-assisted detection of the indicated autophagic markers. $\beta$-actin levels were monitored to ensure equal lane loading. (d and e) MCA205 cells stably transfected with a plasmid-encoding tPANX1 or the corresponding empty vector were left untransfected (UNT) or transiently transfected with a non-targeting siRNA (siUNR) or with the indicated autophagy-modulatory siRNAs for $48 \mathrm{~h}$, and then exposed or not to cumate for additional $48 \mathrm{~h}$. Eventually, cells were subjected to the cytofluorometric detection of YO-PRO-1 and DAPI uptake, whereas ATP levels in culture supernatants were quantified by means of a luciferase-based assay. Quantitative data (means \pm S.E.M., $n=3$ ) are reported. ns, non-significant (Student's t-test) as compared with UNT cells treated with cumate only

cumate-inducible promoter. ${ }^{45}$ The administration of cumate to tPANX1-transfected, but not to control, MCA205 cells rendered them permeable to the small fluorescent dye YO-PRO-1, but not to the vital dye DAPI (Figure $3 \mathrm{c}$ ), in line with the previously reported capacity of tPANX1 to promote a selective permeabilization of the plasma membrane. ${ }^{32-34}$ The expression of tPANX1 drove a YO-PRO-1 influx coupled to an ATP efflux that could be inhibited in a dose-dependent manner by monensin and DIDS but not by Y-27632, blebbistatin and Z-VAD-fmk (Figures $3 d$ and e), confirming the specificity of this panel of inhibitors.

The depletion of the essential autophagic factor ATG5 failed to affect the translocation of endogenous PANX1 to the surface of chemotherapy-treated U2OS cells (Figure 4a). Along with similar lines, CT26 cells stably depleted of Atg5 or Atg7 normally accumulated YO-PRO-1 upon exposure to OXA in vitro (Figure 4b). The expression of tPANX1 as triggered by cumate did not stimulate autophagy, as evaluated by the electrophoretic mobility of the autophagic factor $\mathrm{LC}^{46}$ (Figure $4 \mathrm{C}$ ) and by assessing the redistribution of a green fluorescent protein (GFP)-LC3 chimera into cytoplasmic dots (data not shown). Moreover, the depletion of ATG5, ATG7 and Beclin 1 (BCN1, another protein with a prominent role in autophagy) failed to affect YO-PRO-1 influx into and ATP efflux from MCA205 cells expressing tPANX1 in response to cumate (Figures $4 d$ and e). Similarly, genetic inhibition of autophagy did not prevent the influx of
YO-PRO-1 and the secretion of ATP as triggered in U2OS cells by the constitutive overexpression of tPANX1 (Supplementary Figure 8). Thus, there is no direct causeeffect relationship between the opening of PANX1 channels and autophagy.

Altogether, these results indicate that PANX1 channels, which are required for ICD, mediate ATP secretion in an autophagy-independent manner.

Essential role for LAMP1 in ICD-associated ATP release. To gain further insights into the autophagy-dependent mechanisms accounting for the ICD-associated secretion of ATP, we monitored the subcellular localization of ATP. To this aim, U2OS cells were co-stained with the ATP-sensitive dye quinacrine and organelle-specific fluorochromes, then analyzed by confocal fluorescence microscopy in baseline conditions or upon exposure to OXA. Only a minority of quinacrine-stained vesicles co-localized with mitochondrion- or ER-specific markers, both in untreated and in chemotherapy-treated U2OS cells. Conversely, in control conditions, most quinacrine-labeled vesicles were co-stained by a lysosome-specific acidophilic dye (LysoTracker Red). After the administration of OXA, the colocalization between quinacrine and either LysoTracker Red or DQ Red BSA (a marker of degradative cell compartments) was significantly reduced (Figures $5 \mathrm{a}$ and $\mathrm{b}$ and Supplementary Figure 9). Moreover, the destruction of lysosomes by means of glycyl-L-phenylalanine 2-naphthylamide, a lysosome-disrupting 

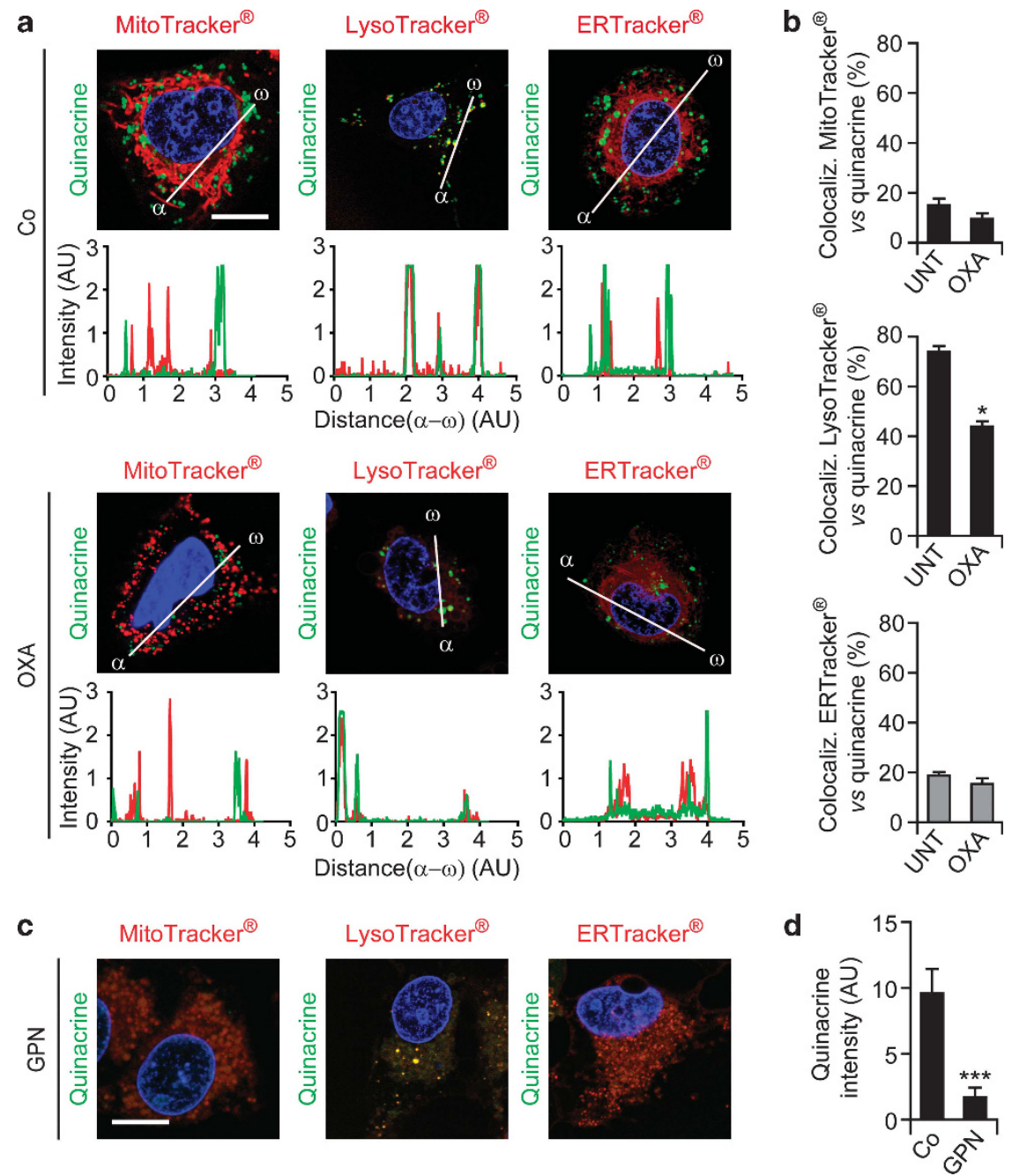

Figure 5 Co-localization between ATP-containing vesicles and lysosomes. (a-d) Human osteosarcoma U2OS cells were maintained in control conditions (Co), incubated with $600 \mu \mathrm{M}$ oxaliplatin (OXA) for $18 \mathrm{~h}$ ( $\mathbf{a}$ and $\mathbf{b}$ ) or with $300 \mu \mathrm{M}$ glycyl---phenylalanine 2-naphthylamide (GPN) for $15 \mathrm{~min}$ (c and $\mathbf{d}$ ), and then co-stained for the fluorescence microscopy-assisted visualization of nuclei (with Hoechst 33342, blue fluorescence), ATP-containing vesicles (with quinacrine, green fluorescence) and mitochondria (with MitoTracker Red), lysosomes (with LysoTracker Red) or the endoplasmic reticulum (with ERTracker Red). Representative images (scale bar $=10 \mu \mathrm{m}$ ), representative fluorescent signals along randomly assigned $\alpha \rightarrow \omega$ axes and quantitative data (means \pm S.E.M., $n=3$ ) are reported. ${ }^{*} P<0.05,{ }^{* * *} P<0.001$ (Student's $t$-test) as compared with cells maintained in Co conditions

cathepsin C substrate, ${ }^{47}$ drastically reduced the intensity of quinacrine fluorescence, while leaving the ER and mitochondria morphologically intact (Figures $5 \mathrm{c}$ and d). Further supporting the preference of quinacrine for lysosomes, untreated U2OS cells exhibited a preponderant co-localization between quinacrine and an LAMP1-red fluorescent protein (RFP) fusion protein, ${ }^{48}$ which is specifically found in lysosomes, and such colocalization was reduced upon exposure to MTX and OXA but not to rapamycin (Figures 6a and b). Concomitantly, OXA, MTX and rapamycin all promoted the co-localization of quinacrine with a RFP-LC3 chimera ${ }^{49}$ that marks autophagosomes and autolysosomes (Figures $6 \mathrm{c}$ and d). Of note, the fraction of ATPrepleted (that is quinacrine ${ }^{+}$) LAMP1-RFP ${ }^{+}$vesicles decreased upon the administration of OXA (Figures $6 e$ and f). This phenomenon was exacerbated by the siRNA-mediated depletion of ATG5, indicating that lysosomes require autophagy for preserving luminal ATP levels.
Taken together, these findings suggest that lysosomes and autophago(lyso)somes directly participate in the trafficking and/or maintenance of the intracellular ATP pool. Further substantiating this possibility, we found that the depletion of LAMP1, a lysosomal protein that is dispensable for autophagy, ${ }^{50}$ abolishes ATP secretion by U2OS cells exposed to MTX or OXA (Figures 7a-c). The knockdown of vesicle-associated membrane protein 1 (VAMP1, also known as synaptobrevin 1), which is known to have a non-redundant role in exocytosis, ${ }^{51}$ also inhibited ATP release in this system, similar to the depletion of ATG5 and BCN1 (Figure 7b). Importantly, MTX or OXA induced the translocation of LAMP1 to the cell surface, as determined by immunofluorescence microscopy upon the staining of non-permeabilized cells with a specific antibody. The translocation of LAMP1 to the surface of MTX- or OXA-treated, yet viable $\left(\mathrm{DAPI}^{-}\right)$, U2OS cells, which could be detected either as a standalone 

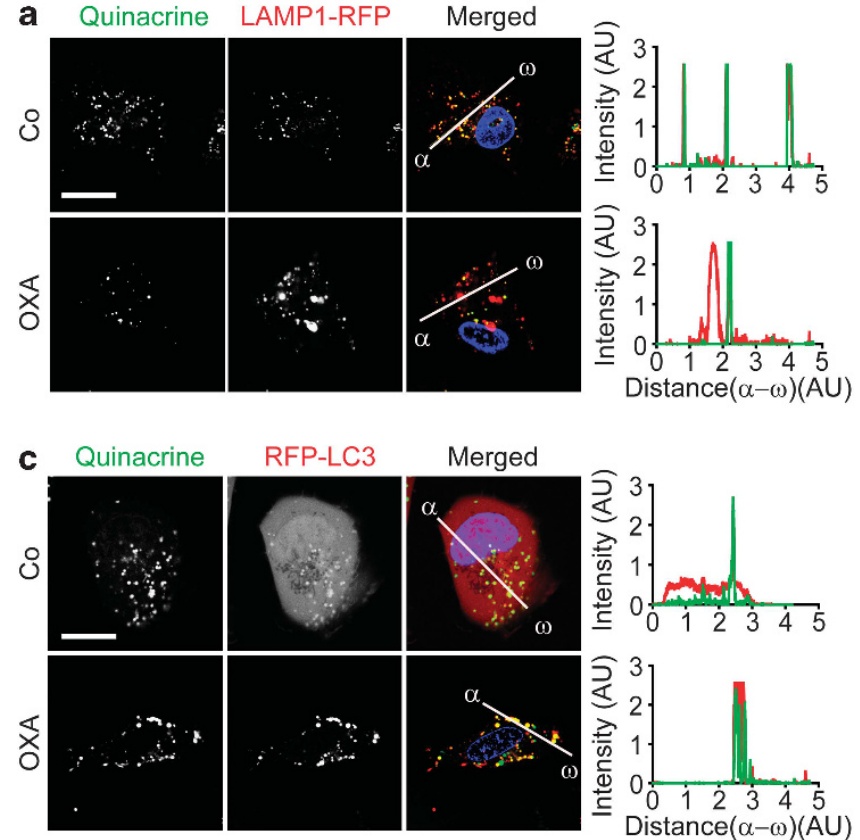

e
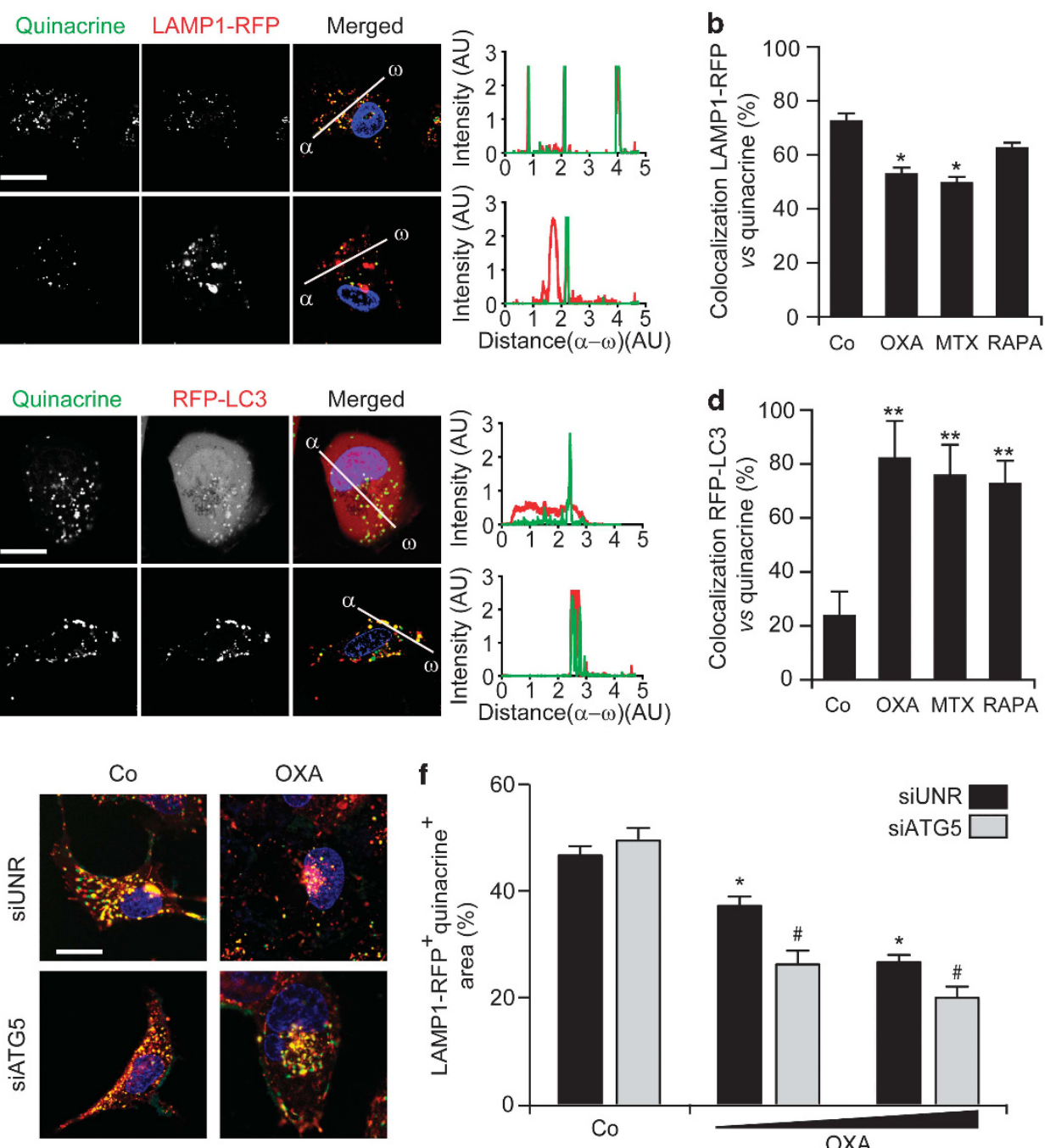

Figure 6 Chemotherapy-induced ATP redistribution into autophago(lyso)somes. (a-d) Human osteosarcoma U2OS cells stably transfected with a plasmid coding for a LAMP1-RFP (a and b) or a RFP-LC3 (c and d) chimera were maintained in control conditions (Co) or treated with $600 \mu \mathrm{M}$ oxaliplatin (OXA), $4 \mu \mathrm{M}$ mitoxantrone (MTX) or $1 \mu \mathrm{M}$ rapamycin (RAPA) for $18 \mathrm{~h}$, and then processed for the fluorescence microscopy-assisted visualization of nuclei (with Hoechst 33342 , blue fluorescence), ATP-containing vesicles (with quinacrine, green fluorescence) and LAMP1-RFP (a and b) or RFP-LC3 (c and d; both emitting in red). Representative images (scale bar $=10 \mu \mathrm{m}$ ), representative fluorescent signals along randomly assigned $\alpha \rightarrow \omega$ axes and quantitative data (means \pm S.E.M., $n=3$ ) are reported. ${ }^{*} P<0.05$, ${ }^{* \star} P<0.01$ (Student's $t$-test) as compared with cells maintained in Co conditions. (e and f) U2OS cells stably expressing LAMP1-RFP were transfected with a non-targeting siRNA (siUNR) or with a siRNA targeting ATG5 for $48 \mathrm{~h}$, and then were either maintained in Co conditions or treated with 300 or $600 \mu \mathrm{M}$ OXA for additional $18 \mathrm{~h}$. Finally, cells were processed for the fluorescence microscopy-assisted visualization of nuclei (with Hoechst 33342, blue fluorescence), ATP-containing vesicles (with quinacrine, green fluorescence) and LAMP1RFP (emitting in red). Representative images (scale bar $=10 \mu \mathrm{m}$ ) and quantitative data on the $\%$ of the LAMP1-RFP ${ }^{+}$intracellular surface not stained with quinacrine (means \pm S.E.M., $n=3$ ) are reported. ${ }^{*} P<0.05$ (Student's $t$-test) as compared with siUNR-transfected cells maintained in Co conditions; ${ }^{\#} P<0.05$ (Student's $t$-test) as compared with siUNR-transfected cells treated with the same dose of OXA

phenomenon or combined with the exposure of PS, was inhibited by Z-VAD-fmk but neither by ROCK1 inhibitors such as $\mathrm{Y}-27632$ and $\mathrm{H} 1152$ nor by blebbistatin (Figures 7d and e and Supplementary Figure 10). Accordingly, the knockdown of PANX1 (but not that of ROCK1, ATG5, ATG7 or BCN1) significantly reduced the LAMP1 exposure on the surface of U2OS cells responding to OXA (Figure 7f). Similarly, the depletion of LAMP1 inhibited the OXA-induced translocation of PANX1 to the outer leaflet of the plasma membrane (Figure $7 \mathrm{~g}$ ), pointing to an unexpected link between PANX1 activation and lysosomal traffic.

Altogether, these results indicate that a pool of intracellular ATP distributes between lysosomes and autolysosomes and suggest that the lysosomal protein LAMP1 has an essential role in ICD-associated ATP secretion, correlating with its caspase- and PANX1-dependent translocation to the cell surface.

\section{Discussion}

The findings presented in this paper reveal an unexpected complexity in the mechanisms that underpin the secretion of ATP by cancer cells undergoing ICD.

Our results are compatible with the widely accepted model suggesting that the cleavage of PANX1 by caspases is required for its activation. ${ }^{32-34}$ Surprisingly, however, the 


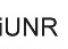

TG5

\section{AMP1}

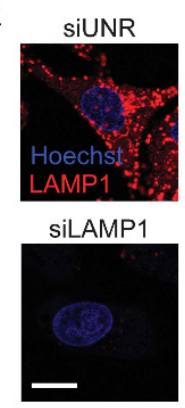

d

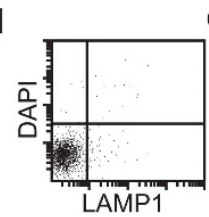

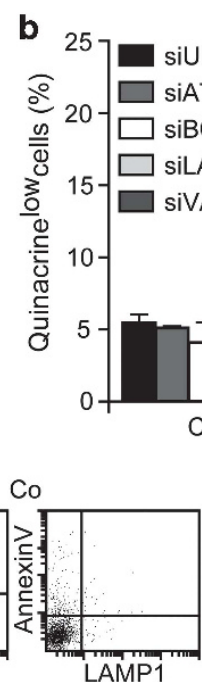

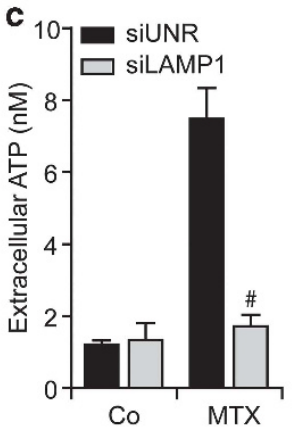

OXA
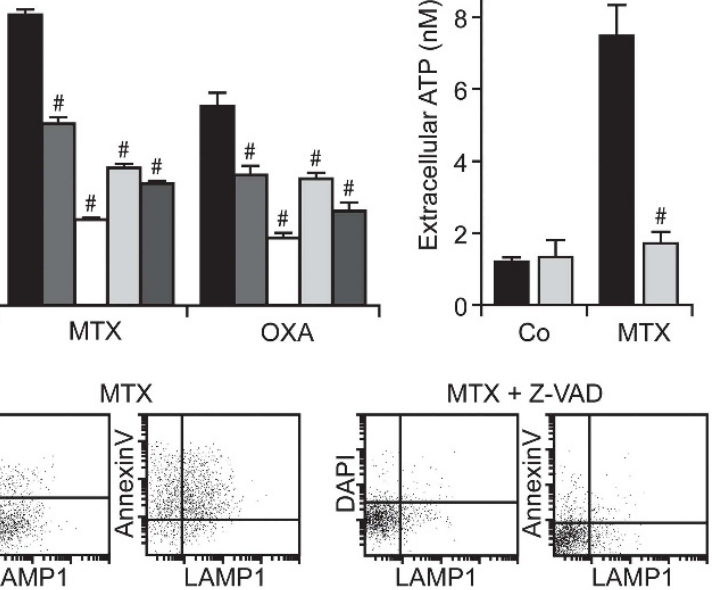

Z-VAD

e

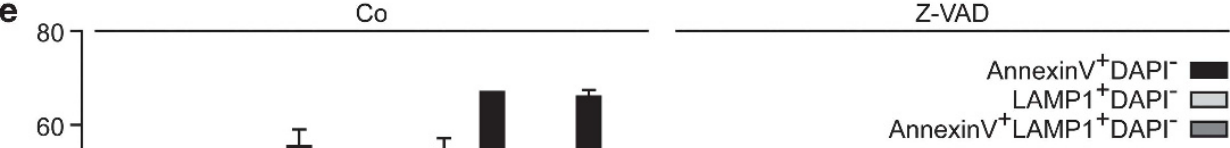

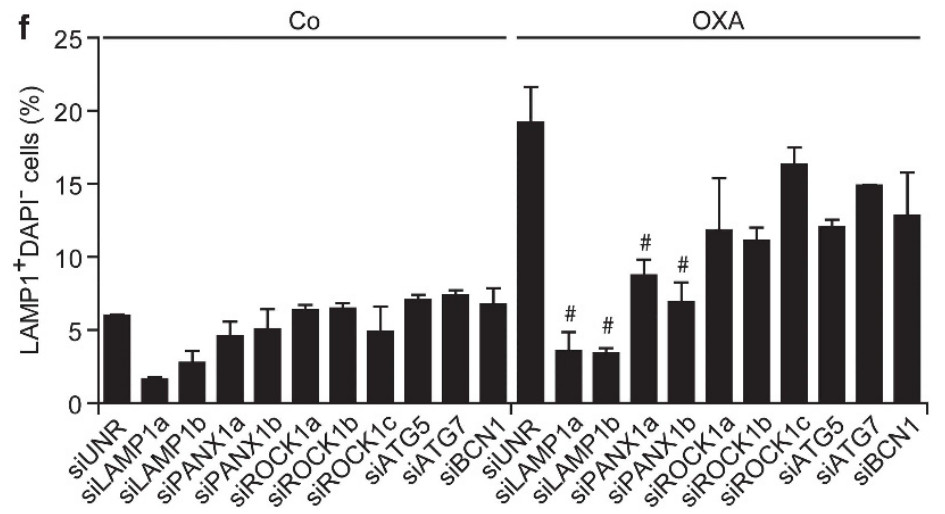

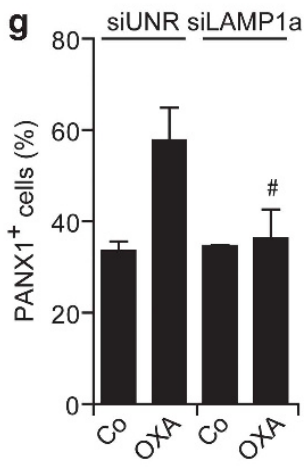

Figure 7 Lysosomes and autophago(lyso)somes participate in the trafficking and/or in the maintenance of the intracellular ATP pool. (a-c) Human osteosarcoma U2OS cells were transfected with a non-targeting siRNA (siUNR) or with the indicated siRNAs for $48 \mathrm{~h}$, and then maintained in control conditions (Co) or treated with $4 \mu \mathrm{M}$ mitoxantrone (MTX) or $300 \mu \mathrm{M}$ oxaliplatin (OXA) for additional $18 \mathrm{~h}$. Finally, cells were processed for the fluorescence microscopy-assisted visualization of nuclei (with Hoechst 33342, blue fluorescence), ATP-containing vesicles (with quinacrine, green fluorescence) or LAMP1 (revealed with an secondary antibody emitting in red) ( $\mathbf{a}$ and $\mathbf{b}$ ), and ATP levels in culture supernatants assessed by a luciferase-based test (c). Representative images (scale bar $=10 \mu \mathrm{m}$ ) and quantitative data (means \pm S.E.M., $n=3$ ) are reported. ${ }^{\#} P<0.05$ (Student's $t$-test), as compared with siUNR-transfected cells treated with MTX or OXA, as appropriate. (d and e) U2OS cells were maintained in Co conditions or treated with $1 \mu \mathrm{M}$ rapamycin (RAPA), 150 or $300 \mu \mathrm{M}$ OXA, or 2 or $4 \mu \mathrm{M} \mathrm{MTX,} \mathrm{alone} \mathrm{or} \mathrm{in} \mathrm{combination} \mathrm{with} 50 \mu \mathrm{M}$ Z-VAD-fmk (Z-VAD), for $18 \mathrm{~h}$. Thereafter, LAMP1 exposure (with a LAMP1-specific antibody revealed in red), phosphatidylserine externalization (with FITC-conjugated AnnexinV, green fluorescence) and cell deathassociated plasma membrane permeabilization (with DAPI, blue fluorescence) were monitored by flow cytometry. Quantitative data (means \pm S.E.M., $n=3$ ) are reported. ${ }^{\#} P<0.05$ (Student's $t$-test), as compared with cells treated with an equivalent concentration of OXA or MTX in the absence of Z-VAD. (f and $\mathbf{g}$ ) U2OS cells were transfected with siUNR or with the indicated siRNAs for $48 \mathrm{~h}$, and then either left untreated (Co) or exposed to $300 \mu \mathrm{M}$ OXA for $18 \mathrm{~h}$. Finally, either LAMP1 exposure (with a LAMP1specific antibody revealed in red), and cell death-associated plasma membrane permeabilization (with DAPI, blue fluorescence) was assessed by cytofluorometry (f), or PANX1 exposure was determined by immunofluorescence microscopy $(\mathbf{g})$. Quantitative data (means \pm S.E.M., $n=3$ ) are reported. ${ }^{*} P<0.05$ (Student's $t$-test), as compared with siUNR-transfected cells treated with OXA 


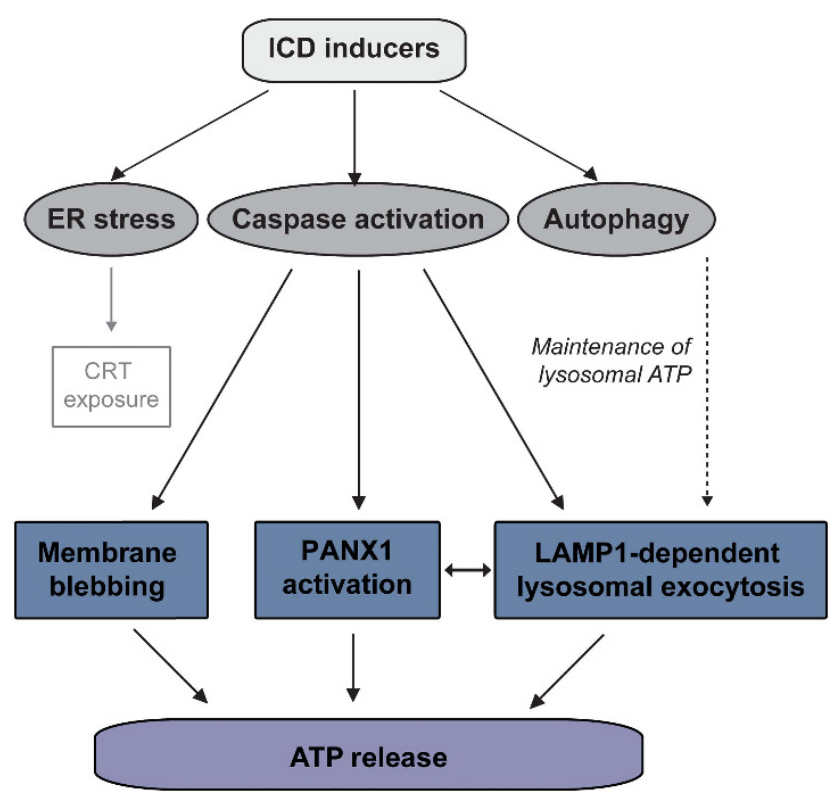

Figure 8 Mechanism of ATP secretion during immunogenic cell death (ICD). ICD inducers such as mitoxantrone and oxaliplatin simultaneously activate various cytoprotective and cytotoxic signaling pathways, including the endoplasmic reticulum (ER) stress response, which is responsible for the exposure of calreticulin (CRT) on the cell surface; the caspase cascade, which - among various proapoptotic functions - mediates cell blebbing as well as the activation of pannexin 1 (PANX1); and autophagy, which is required for the trafficking/maintenance of vesicular ATP pools. A complex crosstalk among all these signaling modules appears to be required for the ICD-associated release of ATP

broad-spectrum caspase inhibitor Z-VAD-fmk not only did reduce the channel activity of PANX1, but also affected its subcellular localization. The amount of immunofluorescencedetectable PANX1 increased at the surface of cells exposed to ICD inducers, and this effect was strictly caspase dependent. Nonetheless, the knockdown of either ATG5 or ATG7, an autophagy-inhibitory manipulation that strongly inhibits ICD-associated ATP secretion, failed to influence the surface exposure and activation state of PANX1. This held true in two distinct models, that is, the caspase-dependent activation of PANX1 as triggered by ICD inducers and the inducible expression of an artificially truncated variant of PANX1 (tPANX1), de facto mimicking the effect of caspase activation. In both these models, the inhibition of autophagy failed to prevent the selective increase in the permeability of the plasma membrane to the small fluorophore YO-PRO- 1.

It is generally assumed that ATP freely distributes in the cytosol at a concentration of $1-10 \mathrm{mM}^{52}$ and that the opening of channels across the plasma membrane would allow for its passive diffusion into the extracellular space. ${ }^{53}$ Our observations are at odds with this view. Indeed, the caspase-induced formation as well as the transfection-enforced expression of tPANX1 increased the permeability of the plasma membrane to YO-PRO-1 in both wild-type and autophagy-deficient cells, yet ATP was efficiently released only by the former. As the total amount of intracellular ATP is not reduced in autophagydeficient cells, neither in baseline conditions nor upon exposure to chemotherapy, ${ }^{23}$ these results imply that the sole presence of open PANX1 channels is not sufficient for ATP release.
In line with this notion, we found that a substantial fraction of intracellular ATP is present in cytoplasmic vesicles rather than in the cytosol, explaining why it cannot diffuse freely across open PANX1 channels. ATP-containing vesicles turned out to stain positively for lysosomal markers including LAMP1, especially in baseline conditions, an observation that has previously been reported for several cell types. ${ }^{35,54}$ In response to chemotherapy, approximately half of ATP-containing cytoplasmic vesicles maintained their colocalization with LAMP1, yet most of them became positive for RFP-LC3, indicating that in these conditions ATP redistributes to autophagosomes and autolysosomes.

The depletion of LAMP1 as well as that of the lysosomal SNAP receptor (SNARE) VAMP1 and other SNAREs involved in exocytosis (such as VAMP7) ${ }^{30}$ inhibited ICD-associated ATP release in vitro, supporting the notion that lysosomal exocytosis would provide a major contribution to this process. Indeed, a substantial fraction of cells responding to chemotherapy in vitro exposed LAMP1 on the cell surface as it externalized PS residues, in line with the hypothesis that lysosomes fuse with the plasma membrane during the apoptotic process. ${ }^{55}$ In this setting, the depletion of essential autophagic factors including AT5, ATG7 and BCN1 failed to affect the translocation of LAMP1 to the outer leaflet of the plasma membrane, meaning that autophagy is not required for lysosomal exocytosis. In sharp contrast, the knockdown of PANX1 prevented LAMP1 from translocating to the plasma membrane, and vice versa LAMP1 depletion interfered with the externalization of PANX1. Moreover, both PANX1 and LAMP1 were unable to translocate to the plasma membrane in the presence of the broad-spectrum caspase inhibitor Z-VAD-fmk. Altogether, these results suggest that lysosomal exocytosis is essential for the ICD-associated secretion of ATP and that PANX1 contributes to this process through yetto-be determined mechanisms.

Interestingly, the inhibition or depletion of ROCK1 and myosin II reduced the release of ATP from cells exposed to ICD inducers. Thus, the apoptotic blebbing of the plasma membrane appears to be required for ICD-associated ATP secretion, yet could not be connected to any other mechanistic module involved in this process. Indeed, blebbing inhibitors failed to interfere with PANX1 translocation/activation, autophagy and lysosomal exocytosis. Vice versa, the inhibition of PANX1, autophagy and lysosomal exocytosis did not affect blebbing. Hence, blebbing may constitute the very last step in the cascade of events that mediate the ICD-related secretion of ATP, operating downstream of PANX1 channel opening, autophagy and LAMP1 translocation. Formal evidence in support of this hypothesis is still missing.

An important question that remains to be addressed in further detail concerns the contribution of autophagy to ATP release. The suppression of autophagy indeed did not affect PANX1 channel function and the relocalization of LAMP1 to the cell surface. Nonetheless, ATP redistributes to bona fide autophago(lyso)somes in response to ICD inducers, at least in vitro. As a possibility, such an autophagydependent redistribution of ATP may favor the transport of ATP-containing vesicles to the cell periphery, yet is not required for the exocytosis of $\mathrm{LAMP}^{+}{ }^{+}$vesicles that do not contain ATP. 
Altogether, our findings reveal a surprisingly complex pathway that mediates the ICD-associated secretion of ATP (Figure 8), involving molecules that are essential for apparently distinct processes such as autophagy (i.e., ATG5, ATG7 and BCN1), lysosomal exocytosis (i.e., LAMP1, VAMP1), apoptosis (i.e., caspases), membrane blebbing (i.e., ROCK1, myosin II) and plasma membrane permeabilization (i.e., PANX1). Molecular defects in any of these systems are sufficient to abolish the in of ATP from cells exposed to ICD inducers, meaning that alterations in autophagy, lysosomal function, caspase activation, membrane blebbing and plasma membrane permeabilization might all contribute to the development of therapy-resistant neoplasms.

\section{Materials and Methods}

Chemical, cell lines and culture conditions. Unless otherwise indicated, media, antibiotics and supplements for cell culture were purchased from Gibco-Life Technologies (Carlsbad, CA, USA), plasticware from Corning B.V. Life Sciences (Schiphol-Rijk, the Netherlands) and chemicals from Sigma-Aldrich (St. Louis, MO, USA). H-1152 was purchased from Alexis Biochemicals (San Diego, CA, USA), benzyloxycarbonyl-Val-Ala-Asp(OMe)-fluoromethylketone (Z-VAD-fmk) from Bachem (Bubendorf, Switzerland), G418 sulfate from Calbiochem (San Diego, CA, USA), and puromycin and zeocin from Invivogen (San Diego, CA, USA). Murine colon carcinoma CT26 cells (MHC Class I haplotype $\mathrm{H}-2^{\mathrm{d}}$, syngenic to BALB/c mice), murine fibrosarcoma MCA205 cells (MHC Class I haplotype $\mathrm{H}-2^{\mathrm{b}}$, syngenic to $\mathrm{C} 57 \mathrm{BI} / 6$ mice) and their derivatives were cultured in RPMI 1640 medium supplemented with 10\% heat-inactivated fetal bovine serum, $10 \mathrm{mM}$ HEPES buffers, $10 \mathrm{U} / \mathrm{ml}$ penicillin sodium and $10 \mu \mathrm{g} / \mathrm{ml}$ streptomycin sulfate, whereas human osteocarcoma U2OS cells and their derivatives were maintained in DMEM supplemented as above. U2OS cells stably co-expressing GFP-LC3 or HMGB1-GFP ${ }^{56}$ were maintained under $200 \mu \mathrm{g} / \mathrm{ml}$ G418 selective pressure. U2OS cells engineered for the stable expression of a CRT-GFP fusion ${ }^{17}$ were cultured in the continuous presence of $100 \mu \mathrm{g} / \mathrm{ml}$ zeocin Control (SCR), Atg5 $5^{\mathrm{KD}}$ and $\operatorname{Atg}^{\mathrm{KD}}$ MCA205 cells $^{23}$ were maintained under $5 \mu \mathrm{g} / \mathrm{ml}$ puromycin selection. To obtain cells stably expressing GFP-LC3, RFPLC3, H2B-RFP or LAMP1-RFP chimeras, the corresponding constructs (Addgene, Cambridge, MA, USA) were transfected into U2OS cells using the Lipofectamine 2000 transfection reagent (Life Technologies, Carlsbad, CA, USA), following the manufacturer's recommendations. Transfected cells were then maintained under $500 \mu \mathrm{g} / \mathrm{ml} \mathrm{G418} \mathrm{selection,} \mathrm{and} \mathrm{GFP-} \mathrm{or} \mathrm{RFP-expressing} \mathrm{cells} \mathrm{were} \mathrm{cloned} \mathrm{by}$ cytofluorometry and amplified.

RNA interference and transfections. siRNAs specific for murine Atg5 (sense $5^{\prime}$-CAUCAACCGGAAACUCAUdTdT- $3^{\prime}$ ), murine Atg7 (sense $5^{\prime}$-AGUUUC CAGUCCGUUGAAdTdT-3'), human ATG5 (sense $5^{\prime}$-CCUUUGGCCUAAGAAGA AAdTdT-3'), human ATG7 (sense $5^{\prime}$-CGACUUGUUCCUUACGGAAdTdT-3') human BCN1 (sense $5^{\prime}$-CAGUGGAUCUAAAUCUCAAdTdT-3'), human LAMP1 (siLAMP1a sense 5'-CGAGAAAUGCAACACGUUAdTdT-3'; siLAMP1b sense, 5'-GGAAUCCAGUUGAAUACAAdTdT-3'), human ROCK1 (siROCK1a sense 5'-GCCGCCGGGACCCAACUAUdTdT-3'; siROCK1b sense 5'-CAGCCAUCACU AUCAAGAUdTdT-3'; siROCK $1 \mathrm{C}$ sense $5^{\prime}$-GACUAUACAAAACUAUUUUdTdT- $3^{\prime}$ ) and human VAMP1 (sense $5^{\prime}$-GGACAUCAUGCGUGUGAAUdTdT-3'), as well as a siRNA unrelated to the murine and human genome (siUNR, sense $5^{\prime}$-GCC GGUAUGCCGGUUAAGUdTdT-3 $\left.3^{5}\right)^{57}$ were purchased from Sigma-Proligo (Woodlands, TX, USA). In addition, pre-designed commercial siRNAs specific for murine Bcn1 (sc-29798) and human SLC17A9 (best known as VNUT; sc-72740) were obtained from Santa Cruz Biotechnology (Santa Cruz, CA, USA). Cells (at $30-40 \%$ confluence) were transfected with siRNAs by means of the HiPerFect transfection reagent (Qiagen, Hilden, Germany) as instructed per manufacturer's instructions, and were used in experimental determinations no earlier than $48 \mathrm{~h}$ after transfection, when target downregulation was monitored by immunoblotting (see below). Transient plasmid transfections were carried out by means of the Lipofectamine 2000 transfection reagent.

Inducible expression of tPANX1. The sequence coding for PANX residues 1-371 coupled to a Flag tag (DYKDDDDK) was amplified from a
pEBB-Flag vector encoding the PANX1 site B mutant (kindly provided by Dr. Kodi $S$ Ravichandran, Carter Immunology Center, University of Virginia, Charlottesville, VA, USA) by means of the following primers: $5^{\prime}$-ATGCTAGCATGGCCATCGCTC AACTG- $3^{\prime}$ and $5^{\prime}$-CAATTTCGAAGTCGGTACCTCACTTGTCATCG- $3^{\prime}$. The PCR product was digested with BstBI and Nhel-HF (New England Biolabs, Ipswich, MA, USA) and inserted into the PCDH-CuO-MCS-EF1-CymR-T2A-Puro All-in-one inducible lentiviral vector (System Biosciences, Mountain View, CA, USA), fused to a hemagglutinin tag. Pseudotyped lentiviral particles coding for tPANX1 were produced with the ViraPower Lentiviral Packaging Mix (Life Technologies), following standard protocols. MCA205 cells were infected with lentiviral vectors at a multiplicity of infection of five in complete culture medium supplemented with $5 \mu \mathrm{g} / \mathrm{ml}$ polybrene overnight. The lentivirus-containing medium was removed and infected cells were allowed to recover in fresh medium for $24 \mathrm{~h}$ before the addition of $5 \mu \mathrm{g} / \mathrm{ml}$ puromycin as a selection antibiotic. One week later, single-cell clones were isolated on a FACSVantage cytofluorometer (Becton Dickinson, San José, CA, USA), amplified and screened for constitutive Flag or hemagglutinin (tPANX1) expression.

Immunoblotting. For immunoblotting, approximately $1 \times 10^{6}$ cells were washed with cold PBS and lysed following standard procedures. ${ }^{58}$ To this aim, $50 \mu \mathrm{g}$ of proteins were separated according to molecular weight on NuPAGE Novex Bis-Tris 4-12\% pre-cast gels (Life Technologies) and electrotransferred to nitrocellulose membranes (Bio-Rad, Hercules, CA, USA). Unspecific binding sites were blocked by incubating membranes for $1 \mathrm{~h}$ in $0.05 \%$ Tween-20 (v/v in TBS) supplemented with $5 \%$ non-fat powdered milk or bovine serum albumin, followed by overnight incubation at $4{ }^{\circ} \mathrm{C}$ with primary antibodies specific for: ATG5 (Sigma-Aldrich), ATG7 (Sigma-Aldrich), BCN1 (Santa-Cruz Biotechnology), GAPDH (Millipore, Billerica, MA, USA), LC3B (Cell Signaling Technology, Danvers, MA, USA), p62 (Abnova, Taipei, Taiwan), ROCK1 (Biosciences, San Diego, CA, USA) or VNUT (Santa-Cruz Biotechnology). Equal lane loading was monitored by probing membranes with antibodies specific for $\beta$-actin (MilliporeChemicon International, Temecula, CA, USA) or GAPDH (Millipore-Chemicon International). Primary antibodies were detected with appropriate horseradish peroxidase-labeled secondary antibodies (Southern Biotechnologies Associates, Birmingham, UK) and were revealed on a ImageQuant LAS 4000 softwareassisted imager (GE Healthcare, Piscataway, NJ, USA) upon incubation with the SuperSignal West Pico reagent (Thermo Fisher Scientific, Rockford, IL, USA) or the ECL Plus Western Blotting Detection System (GE Healthcare).

Cell death and membrane permeability assays. Apoptotic cell death was monitored by virtue of PS exposure and the uptake of the vital dye DAPI. ${ }^{59,60}$ To this aim, cells were washed twice with PBS and resuspended in $1 \times$ binding buffer supplemented with PE- or fluorescein isothiocyanate (FITC)-conjugated AnnexinV (BD Biosciences, Franklin Lakes, NJ, USA) and DAPI, as per manufacturer's instructions. For the assessment of plasma membrane permeability, cells were incubated in culture medium supplemented with $1 \mu \mathrm{M}$ YO-PRO-1 (Molecular Probes-Life Technologies, Eugene, CA, USA) for $30 \mathrm{~min}$ at $37^{\circ} \mathrm{C}$. Cytofluorometric determinations were carried out on a Gallios cytofluorometer (Beckman Coulter, Brea, CA, USA) and data were analyzed by means of the Kaluza software v. 1.1 (Beckman Coulter) upon gating on events displaying normal forward scatter and side scatter parameters.

Labeling of vesicular ATP. Vesicular ATP was detected by fluorescence microscopy or cytofluorometry, as previously described. ${ }^{29}$ For fluorescence microscopy studies, cells were loaded with $5 \mu \mathrm{M}$ quinacrine in Krebs-Ringer solution ( $125 \mathrm{mM} \mathrm{NaCl}, 5 \mathrm{mM} \mathrm{KCl}, 1 \mathrm{mM} \mathrm{MgSO}_{4}, 0,7 \mathrm{mM} \mathrm{KH}_{2} \mathrm{PO}_{4}, 2 \mathrm{mM} \mathrm{CaCl}_{2}$, $6 \mathrm{mM}$ glucose and $25 \mathrm{mM}$ HEPES buffer, $\mathrm{pH} 7.4$ ) for $30 \mathrm{~min}$ at $37^{\circ} \mathrm{C}$. Thereafter, cells were co-stained for $10 \mathrm{~min}$ with $1 \mu \mathrm{g} / \mathrm{ml}$ propidium iodide $(\mathrm{PI})$ and $10 \mu \mathrm{M}$ Hoechst 33342 (both from Molecular Probes-Life Technologies), rinsed with Krebs-Ringer solution and examined on a confocal SPE microscope equipped with Apochromat $63 \times 1.3 \mathrm{NA}$ and $40 \times 1.15 \mathrm{NA}$ oil immersion objectives (Leica, Wetzlar, Germany). The open-source software Image $J$ (freely available at http://rsbweb.nih.gov/ij/) was used to determine co-localization profiles for quantitative studies. The percentage of co-localization between LAMP1-RFP or RFP-LC3 and quinacrine was calculated by means of the Metamorph software $v$. 6.2r4 (Universal Imaging Corp., Detroit, MI, USA). For cytofluorometric determinations, cells were loaded with $1 \mu \mathrm{M}$ quinacrine as described above, rinsed and resuspended in $1 \mu \mathrm{g} / \mathrm{ml} \mathrm{PI}$ or, alternatively, loaded with $30 \mu \mathrm{M}$ BodipyATP (Life Technologies), following the manufacturer's recommendations. 
In both cases, fluorescence was acquired on a FACSVantage cytofluorometer and first-line statistical analyses were performed by means of the CellQuest software (Becton Dickinson) upon gating on events displaying normal forward scatter and side scatter parameters.

Cells sorting. One million five hundred thousand U2OS cells were stained with $150 \mathrm{nM}$ tetramethylrhodamine methyl ester (TMRM, from Molecular ProbesLife Technologies) for $30 \mathrm{~min}$ at $37^{\circ} \mathrm{C}$, washed twice with cold PBS and resuspended in $1 \times$ binding buffer supplemented with FITC-conjugated Annexin V (BD Biosciences) and $10 \mu \mathrm{M} \mathrm{DAPI}$, as per manufacturer's instructions. Cells were sorted in three sub-populations (DAPI ${ }^{-} \mathrm{PS}^{-} \mathrm{TMRM}^{\text {low; }}$; $\mathrm{DAPI}^{-} \mathrm{PS}^{-} \mathrm{TMRM}^{\text {high, }}$; $\mathrm{DAPI}^{-} \mathrm{PS}^{+} \mathrm{TMRM}^{\mathrm{low}}$ ) on a FACSVantage cytofluorometer and then either collected for the assessment of intracellular ATP or maintained in culture for additional $60 \mathrm{~min}$ for the quantification of extracellular ATP (see below).

ATP release assays. Extracellular and intracellular ATP levels were measured by the luciferin-based ENLITEN ATP Assay (Promega, Madison, WI, USA) and ATP assay (Calbiochem) kits, respectively. ATP-driven chemoluminescence was recorded on a FLUOstar OPTIMA FL Plate Reader (BMG Labtech, Offenburg, Germany).

Cell trackers. Cells were grown in Lab-Tek chambered coverglasses (NUNC, Roskilde, Denmark). Upon the removal of the culture medium, cells were incubated with pre-warmed medium containing $500 \mathrm{nM}$ MitoTracker, $500 \mathrm{nM}$ ER-Tracker, $500 \mathrm{nM}$ Lysotracker or $10 \mu \mathrm{g} / \mathrm{ml} \mathrm{DQ}$ Red BSA (all from Molecular Probes-Life Technologies) for $1 \mathrm{~h}$ at $37^{\circ} \mathrm{C}$. Thereafter, cells were rinsed twice with pre-warmed medium, labeled with quinacrine and Hoechst 33342 and processed for fluorescence microscopy, as described above.

Analysis of cell-surface-exposed LAMP1. Cells were washed twice with cold PBS, incubated for 45 min with a PE-conjugated LAMP1-specific antibody (Biolegend, San Diego, CA, USA) or isotype-matched control antibodies (Biolegend) in cold blocking buffer ( $3 \%$ fetal bovine serum in PBS). Upon washing in cold PBS, samples were stained with DAPI and analyzed for the presence of LAMP1 at the surface of viable $\left(\mathrm{DAPI}^{-}\right)$cells on a Gallios cytofluorometer. First-line statistical analyses were performed by means of the Kaluza software v. 1.1.

Study of cell-surface-exposed PANX1. PANX1 exposure was assessed by surface immunostaining coupled to fluorescence microscopy. To this aim, cells grown on Lab-Tek chambered coverglasses were washed in PBS, incubated with blocking buffer containing an anti-PANX1 antibody (Abcam, Cambridge, UK) for $30 \mathrm{~min}$ on ice, washed again and incubated for 30 min with an anti-rabbit Alexa Fluor 488 conjugate (Life Technologies). Finally, samples were fixed with $3.7 \%$ paraformaldehyde and $1 \mu \mathrm{g} / \mathrm{ml}$ Hoechst 33342 (w/v in PBS) and analyzed on a confocal SPE microscope equipped with Apochromat $\times 631.3$ NA and $\times 401.15$ NA oil immersion objectives.

Automated high-content videomicroscopy. Eighty thousand CRTGFP-, HMGB1-GFP-, GFP-LC3-expressing or wild-type U2OS cells seeded in poly-L-lysine-coated 96-well Black/Clear Imaging Plates (BD Biosciences) were pre-treated with compounds from the ICCB Known Bioactives Library (Enzo Life Sciences, Farmingdale, NY, USA) or the US-Drug collection (Microsource Discovery Systems, Gaylordsville, CT, USA) at a final concentration of 30 and $2 \mu \mathrm{M}$, respectively, for $2 \mathrm{~h}$, followed by the administration of $4 \mu \mathrm{M}$ MTX or an equivalent volume of PBS. Twenty-four hours later, four to nine viewfields per well were acquired by means of a BD Pathway 855 imaging station (BD Biosciences) and images were analyzed for GFP-LC3 dots, CRT-GFP granularity, ATP secretion or HMGB1-GFP release by using the AttoVision software v. 1.6 (BD Biosciences).

Animal experiments. Animals were maintained in appropriate pathogen-free conditions and all experiments were conducted in lines with the Federation of European Laboratory Animal Science Association guidelines, were approved by the local Ethics Committee (CEEA IRCIV/ IGR n 26, registered with the French Ministry of Research) and were in compliance with the EU 63/2010 directive. BALB/C (MHC Class I haplotype H-2 $2^{d}$ ) and C57BI/6 (MHC Class I haplotype H-2 mice were obtained from Janvier (Le Genest-Saint-Isle, France) or Harlan (Gannat, France), used between 6 and 20 weeks of age and euthanatized when tumors exceeded $20-25 \%$ of the total body mass.
Anti-tumor vaccination experiments. CT26 cells were treated by $2 \mu \mathrm{M}$ MTX, either alone or upon exposure to $10 \mu \mathrm{M}$ DIDS, $10 \mu \mathrm{M}$ SITS, $30 \mu \mathrm{M} \mathrm{Y}-27632$, $2 \mu \mathrm{M} \mathrm{H}-1115$ or $50 \mu \mathrm{M}$ blebbistatin, and inoculated $24 \mathrm{~h}$ later $\left(3 \times 10^{6}\right.$ in $200 \mu \mathrm{l}$ PBS, pH 7.4) S.c. in the lower flank of 6-week-old female BALB/c mice. Seven days later, $5 \times 10^{5}$ living CT26 cells (in $200 \mu$ l PBS, pH 7.4) were inoculated into the contralateral flank, as previously described. ${ }^{7,8}$ Similarly, MCA205 cells were incubated with $1 \mu \mathrm{M}$ MTX (alone or upon exposure to additional chemicals, as detailed above) for $24 \mathrm{~h}$ and then subcutaneously inoculated $\left(3 \times 10^{5}\right.$ in $200 \mu \mathrm{l}$ PBS, pH 7.4) into the lower flank of 6-week-old C57BI/6 female mice. Seven days later, $3 \times 10^{4}$ untreated MCA205 cells (in $200 \mu \mathrm{l}$ PBS, pH 7.4) were inoculated into the contralateral flank. In both cases, mice were monitored for the appearance of tumors three times a week for 60 days.

Statistical analyses. Each experiment has been repeated at least three times, yielding comparable results. Unless otherwise indicated, figures illustrate quantitative data from one representative experiment (means \pm S.E.M., $n=3$ replicate samples). Data were analyzed by means of Prism v. 5.03 (GraphPad Software, La Jolla, CA, USA) and Excel 2007 (Microsoft, Rockville, MD, USA). Statistical significance was assessed by two-tailed Student's $t$-tests or log-rank tests, as appropriate. In all experiments, $P$-values $<0.05$ were considered as statistically significant.

\section{Conflict of Interest}

The authors declare no conflict of interest.

Acknowledgements. IM is supported by Ligue National contre le Cancer, while JLP is funded by Sidaction and Natixis. GK is supported by the European Commission (ArtForce); Agence National de la Recherche (ANR); Ligue National contre le Cancer (Equipe labellisée); Fondation pour la Recherche Médicale (FRM); Institut National du Cancer (INCa); LabEx Immuno-Oncologie; Fondation de France; Fondation Bettencourt-Schueller; AXA Chair for Longevity Research; Cancéropôle lle-de-France; Paris Alliance of Cancer Research Institutes (PACRI) and Cancer Research for Personalized Medicine (CARPEM).

1. Galluzzi L, Senovilla L, Zitvogel L, Kroemer G. The secret ally: immunostimulation by anticancer drugs. Nat Rev Drug Discov 2012; 11: 215-233.

2. Kroemer G, Galluzzi L, Kepp O, Zitvogel L. Immunogenic cell death in cancer therapy. Annu Rev Immunol 2013; 31: 51-72.

3. Krysko DV, Garg AD, Kaczmarek A, Krysko O, Agostinis P, Vandenabeele P. Immunogenic cell death and DAMPs in cancer therapy. Nat Rev Cancer 2012; 12: 860-875.

4. Vanneman M, Dranoff G. Combining immunotherapy and targeted therapies in cancer treatment. Nat Rev Cancer 2012; 12: 237-251.

5. Galluzzi L, Vitale I, Abrams JM, Alnemri ES, Baehrecke EH, Blagosklonny MV et al. Molecular definitions of cell death subroutines: recommendations of the Nomenclature Committee on Cell Death 2012. Cell Death Differ 2012; 19: 107-120.

6. Kroemer G, Galluzzi L, Vandenabeele P, Abrams J, Alnemri ES, Baehrecke EH et al. Classification of cell death: recommendations of the Nomenclature Committee on Cell Death 2009. Cell Death Differ 2009; 16: 3-11.

7. Casares N, Pequignot MO, Tesniere A, Ghiringhelli F, Roux S, Chaput N et al. Caspase-dependent immunogenicity of doxorubicin-induced tumor cell death. J Exp Med 2005; 202: 1691-1701.

8. Obeid M, Panaretakis T, Joza N, Tufi R, Tesniere A, van Endert $\mathrm{P}$ et al. Calreticulin exposure is required for the immunogenicity of gamma-irradiation and UVC light-induced apoptosis. Cell Death Differ 2007; 14: 1848-1850.

9. Tanaka H, Matsushima H, Nishibu A, Clausen BE, Takashima A. Dual therapeutic efficacy of vinblastine as a unique chemotherapeutic agent capable of inducing dendritic cell maturation. Cancer Res 2009; 69: 6987-6994.

10. Ghiringhelli F, Menard C, Puig PE, Ladoire S, Roux S, Martin F et al. Metronomic cyclophosphamide regimen selectively depletes $\mathrm{CD} 4+\mathrm{CD} 25+$ regulatory $\mathrm{T}$ cells and restores $\mathrm{T}$ and $\mathrm{NK}$ effector functions in end stage cancer patients. Cancer Immunol Immunother 2007; 56: 641-648.

11. Green DR, Ferguson T, Zitvogel L, Kroemer G. Immunogenic and tolerogenic cell death. Nat Rev Immunol 2009; 9: 353-363.

12. Galluzzi L, Kepp O, Kroemer G. Mitochondria: master regulators of danger signalling. Nat Rev Mol Cell Biol 2012; 13: 780-788.

13. Ferguson TA, Choi J, Green DR. Armed response: how dying cells influence T-cell functions. Immunol Rev 2011; 241: 77-88.

14. Tesniere A, Schlemmer F, Boige V, Kepp O, Martins I, Ghiringhelli F et al. Immunogenic death of colon cancer cells treated with oxaliplatin. Oncogene 2010; 29: 482-491. 
15. Schiavoni G, Sistigu A, Valentini M, Mattei F, Sestili P, Spadaro F et al. Cyclophosphamide synergizes with type I interferons through systemic dendritic cell reactivation and induction of immunogenic tumor apoptosis. Cancer Res 2011; 71: 768-778.

16. Senovilla L, Vitale I, Martins I, Tailler M, Pailleret C, Michaud M et al. An immunosurveillance mechanism controls cancer cell ploidy. Science 2012; 337 1678-1684.

17. Menger L, Vacchelli E, Adjemian S, Martins I, Ma Y, Shen S et al. Cardiac glycosides exert anticancer effects by inducing immunogenic cell death. Sci Transl Med 2012; 4: 143ra199.

18. Kepp O, Menger L, Vacchelli E, Adjemian S, Martins I, Ma Y et al. Anticancer activity of cardiac glycosides: at the frontier between cell-autonomous and immunological effects. Oncoimmunology 2012; 1: 1640-1642.

19. Menger L, Vacchelli E, Kepp O, Eggermont A, Tartour E, Zitvogel L et al. Trial watch: cardiac glycosides and cancer therapy. Oncoimmunology 2013; 2: e23082.

20. Vacchelli E, Galluzzi L, Fridman WH, Galon J, Sautes-Fridman C, Tartour E et al. Tria Watch: chemotherapy with immunogenic cell death inducers. Oncoimmunology 2012; 1 : 179-188.

21. Vacchelli E, Senovilla L, Eggermont A, Fridman WH, Galon J, Zitvogel L et al. Trial Watch: chemotherapy with immunogenic cell death inducers. Oncoimmunology 2013; 2: e23510.

22. Obeid M, Tesniere A, Ghiringhelli F, Fimia GM, Apetoh L, Perfettini JL et al. Calreticulin exposure dictates the immunogenicity of cancer cell death. Nat Med 2007; 13: $54-61$.

23. Michaud M, Martins I, Sukkurwala AQ, Adjemian S, Ma Y, Pellegatti P et al. Autophagydependent anticancer immune responses induced by chemotherapeutic agents in mice. Science 2011; 334: 1573-1577.

24. Ma Y, Adjemian S, Mattarollo SR, Yamazaki T, Aymeric L, Yang $\mathrm{H}$ et al. Anticancer chemotherapy-induced intratumoral recruitment and differentiation of antigen-presenting cells. Immunity 2013; 38: 729-741.

25. Ayna G, Krysko DV, Kaczmarek A, Petrovski G, Vandenabeele P. Fesus L. ATP release from dying autophagic cells and their phagocytosis are crucial for inflammasome activation in macrophages. PLoS One 2012; 7: e40069.

26. Zitvogel L, Kepp O, Galluzzi L, Kroemer G. Inflammasomes in carcinogenesis and anticancer immune responses. Nat Immunol 2012; 13: 343-351.

27. Vacchelli E, Galluzzi L, Rousseau V, Rigoni A, Tesniere A, Delahaye N et al. Loss-of-function alleles of P2RX7 and TLR4 fail to affect the response to chemotherapy in non-small cell lung cancer. Oncoimmunology 2012; 1: 271-278.

28. Michaud M, Sukkurwala AQ, Martins I, Shen S, Zitvogel L, Kroemer G. Subversion of the chemotherapy-induced anticancer immune response by the ecto-ATPase CD39. Oncoimmunology 2012; 1: 393-395.

29. Martins I, Tesniere A, Kepp O, Michaud M, Schlemmer F, Senovilla L et al. Chemotherapy induces ATP release from tumor cells. Cell Cycle 2009; 8: 3723-3728.

30. Fader CM, Aguilera MO, Colombo MI. ATP is released from autophagic vesicles to the extracellular space in a VAMP7-dependent manner. Autophagy 2012; 8: 1741-1756.

31. Sandilos JK, Chiu YH, Chekeni FB, Armstrong AJ, Walk SF, Ravichandran KS et al. Pannexin 1, an ATP release channel, is activated by caspase cleavage of its pore-associated C-terminal autoinhibitory region. J Biol Chem 2012; 287 11303-11311.

32. Elliott MR, Chekeni FB, Trampont PC, Lazarowski ER, Kadl A, Walk SF et al. Nucleotides released by apoptotic cells act as a find-me signal to promote phagocytic clearance. Nature 2009; 461: 282-286

33. Chekeni FB, Elliott MR, Sandilos JK, Walk SF, Kinchen JM, Lazarowski ER et al. Pannexin 1 channels mediate 'find-me' signal release and membrane permeability during apoptosis. Nature 2010; 467: 863-867.

34. Qu Y, Misaghi S, Newton K, Gilmour LL, Louie S, Cupp JE et al. Pannexin-1 is required for ATP release during apoptosis but not for inflammasome activation. J Immunol 2011; 186 : 6553-6561.

35. Zhang Z, Chen G, Zhou W, Song A, Xu T, Luo $Q$ et al. Regulated ATP release from astrocytes through lysosome exocytosis. Nat Cell Biol 2007; 9: 945-953.

36. Pangrsic T, Potokar M, Stenovec M, Kreft M, Fabbretti E, Nistri A et al. Exocytotic release of ATP from cultured astrocytes. J Biol Chem 2007; 282: 28749-28758.

37. Martins I, Michaud M, Sukkurwala AQ, Adjemian S, Ma Y, Shen S et al. Premortem autophagy determines the immunogenicity of chemotherapy-induced cancer cell death Autophagy 2012; 8: 413-415.

38. Akopova I, Tatur S, Grygorczyk M, Luchowski R, Gryczynski I, Gryczynski Z et al. Imaging exocytosis of ATP-containing vesicles with TIRF microscopy in lung epithelial A549 cells. Purinergic Signal 2012; 8: 59-70.
39. Straight AF, Cheung A, Limouze J, Chen I, Westwood NJ, Sellers JR et al. Dissecting temporal and spatial control of cytokinesis with a myosin II Inhibitor. Science 2003; 299: 1743-1747.

40. Uehata M, Ishizaki T, Satoh $\mathrm{H}$, Ono T, Kawahara T, Morishita T et al. Calcium sensitization of smooth muscle mediated by a Rho-associated protein kinase in hypertension. Nature 1997; 389: 990-994.

41. Coleman ML, Sahai EA, Yeo M, Bosch M, Dewar A, Olson MF. Membrane blebbing during apoptosis results from caspase-mediated activation of ROCK I. Nat Cell Biol 2001; 3: 339-345.

42. Sebbagh M, Renvoize C, Hamelin J, Riche N, Bertoglio J, Breard J. Caspase-3-mediated cleavage of ROCK I induces MLC phosphorylation and apoptotic membrane blebbing. Nat Cell Biol 2001; 3: 346-352.

43. Iglesias R, Locovei S, Roque A, Alberto AP, Dahl G, Spray DC et al. P2 $\times 7$ receptorPannexin1 complex: pharmacology and signaling. Am J Physiol Cell Physiol 2008; 295: C752-C760.

44. Sathe MN, Woo K, Kresge C, Bugde A, Luby-Phelps K, Lewis MA et al. Regulation of purinergic signaling in biliary epithelial cells by exocytosis of SLC17A9-dependent ATPenriched vesicles. J Biol Chem 2011; 286: 25363-25376.

45. Mullick A, Xu Y, Warren R, Koutroumanis M, Guilbault C, Broussau S et al. The cumate gene-switch: a system for regulated expression in mammalian cells. BMC Biotechnol 2006; 6: 43

46. Klionsky DJ, Abdalla FC, Abeliovich H, Abraham RT, Acevedo-Arozena A, Adeli K et al. Guidelines for the use and interpretation of assays for monitoring autophagy. Autophagy 2012; 8: 445-544.

47. Berg TO, Stromhaug E, Lovdal T, Seglen O, Berg T. Use of glycyl-L-phenylalanine 2naphthylamide, a lysosome-disrupting cathepsin C substrate, to distinguish between lysosomes and prelysosomal endocytic vacuoles. Biochem J 1994; 300(Pt 1): 229-236.

48. Sherer NM, Lehmann MJ, Jimenez-Soto LF, Ingmundson A, Horner SM, Cicchetti G et al. Visualization of retroviral replication in living cells reveals budding into multivesicular bodies. Traffic 2003; 4: 785-801.

49. Morselli E, Marino G, Bennetzen MV, Eisenberg T, Megalou E, Schroeder S et al. Spermidine and resveratrol induce autophagy by distinct pathways converging on the acetylproteome. J Cell Biol 2011; 192: 615-629.

50. Gonzalez-Polo RA, Boya P, Pauleau AL, Jalil A, Larochette N, Souquere $S$ et al. The apoptosis/autophagy paradox: autophagic vacuolization before apoptotic death. J Cell Sci 2005; 118: 3091-3102

51. Liu Y, Sugiura $Y$, Lin W. The role of synaptobrevin1/VAMP1 in Ca2 +-triggered neurotransmitter release at the mouse neuromuscular junction. J Physiol 2011; 589: 1603-1618.

52. Ainscow EK, Mirshamsi S, Tang T, Ashford ML, Rutter GA. Dynamic imaging of free cytosolic ATP concentration during fuel sensing by rat hypothalamic neurones: evidence for ATP-independent control of ATP-sensitive K(+ ) channels. J Physiol 2002; 544: $429-445$

53. Li A, Banerjee J, Leung CT, Peterson-Yantorno K, Stamer WD, Civan MM. Mechanisms of ATP release, the enabling step in purinergic dynamics. Cell Physiol Biochem 2011; 28: $1135-1144$.

54. Gonzales E, Julien B, Serriere-Lanneau V, Nicou A, Doignon I, Lagoudakis L et al. ATP release after partial hepatectomy regulates liver regeneration in the rat. J Hepatol 2010; 52 : 54-62.

55. Franz S, Herrmann K, Furnrohr BG, Sheriff A, Frey B, Gaipl US et al. After shrinkage apoptotic cells expose internal membrane-derived epitopes on their plasma membranes. Cell Death Differ 2007; 14: 733-742

56. Martins I, Kepp O, Schlemmer F, Adjemian S, Tailler M, Shen S et al. Restoration of the immunogenicity of cisplatin-induced cancer cell death by endoplasmic reticulum stress. Oncogene 2011; 30: 1147-1158.

57. de La Motte Rouge T, Galluzzi L, Olaussen KA, Zermati Y, Tasdemir E, Robert T et al. A novel epidermal growth factor receptor inhibitor promotes apoptosis in non-small cell lung cancer cells resistant to erlotinib. Cancer Res 2007; 67: 6253-6262.

58. Galluzzi L, Morselli E, Vitale I, Kepp O, Senovilla L, Criollo A et al. miR-181a and miR-630 regulate cisplatin-induced cancer cell death. Cancer Res 2010; 70: 1793-1803.

59. Kepp O, Galluzzi L, Lipinski M, Yuan J, Kroemer G. Cell death assays for drug discovery. Nat Rev Drug Discov 2011; 10: 221-237.

60. Galluzzi L, Zamzami N, de La Motte Rouge T, Lemaire C, Brenner C, Kroemer G. Methods for the assessment of mitochondrial membrane permeabilization in apoptosis. Apoptosis 2007; 12: 803-813 